\title{
Mechanisms to enhance the capacitance beyond the classical limits in capacitors with free-electron-like electrodes
}

\author{
Javier Junquera, ${ }^{1}$ Pablo García-Fernández, ${ }^{1}$ and Massimiliano Stengel ${ }^{2,3}$ \\ ${ }^{1}$ Departamento de Ciencias de la Tierra y Física de la Materia Condensada, Universidad de Cantabria, \\ Cantabria Campus Internacional, Avenida de los Castros s/n, 39005 Santander, Spain \\ ${ }^{2}$ Institut de Ciència de Materials de Barcelona (ICMAB-CSIC), 08193 Bellaterra, Spain \\ ${ }^{3}$ ICREA-Institució Catalana de Recerca i Estudis Avançats, Pg. Lluis Companys, 23, 08010 Barcelona, Spain
}

(Received 12 February 2019; published 12 June 2019)

\begin{abstract}
The negative electronic compressibility refers to the lowering of the chemical potential of a metallic system when the carrier density increases. This effect has often been invoked in the past to explain the enhancement of the capacitance beyond the classical limits in capacitors with two-dimensional electron gases as electrodes. Based on experiments on strongly confined semiconductor quantum wells (QWs), it has been traditionally ascribed to the electron exchange energy as the main driving force. Recent research, however, has revealed that analogous effects can occur in other classes of materials systems, such as polar oxide interfaces, whose characteristics drastically depart from those of the previously considered cases. To rationalize these new results, it is necessary to revisit the established theory of confined electron gases, and test whether its conclusions are valid beyond the specifics of semiconductor-based QWs. Here we find, based on first-principles calculations of jellium slabs, that one must indeed be very careful when extrapolating existing results to other realistic physical systems. In particular, we identify a number of additional, previously overlooked mechanisms (e.g., related to the displacement of the electronic cloud and to the multiband structure of the delocalized gas), that enter into play and become sources of negative capacitance in the weak-confinement regime. Our detailed analysis of these emerging contributions, supported by analytic models and multiple test cases, will provide a useful guidance in the ongoing quest for nanometric capacitors with enhanced performance.
\end{abstract}

DOI: 10.1103/PhysRevB.99.235127

\section{INTRODUCTION}

The constant drive for faster, smaller, cheaper and more powerful microelectronic devices has led to Moore's law [1], the defining paradigm of the global semiconductor industry. Although its validity has firmly held since its formulation in the mid 1960s, often disproving the recurring skepticisms that were raised over the years, the exponential ${ }^{1}$ improvement in computer power summarized in this empirical rule is now coming to an end $[2,3]$. The main culprit is the inevitable shrinking of the electronic components of the integrated circuits, whose size is currently approaching the ultimate physical limit of a single atomic layer. Within this regime, traditional design principles have become unreliable as quantum size effects start to kick in; moreover, Joule heating (e.g., related to parasitic tunneling currents) has become so dramatic as to seriously compromise the device efficiency, or even its functionality.

To illustrate why miniaturization is directly linked to energy waste, it is useful to consider the example of metaloxide-semiconductor field-effect transistors (MOSFET), an ubiquitous device in modern microprocessors. In MOSFETs, the gate metal electrode and the semiconducting channel are

\footnotetext{
${ }^{1}$ According to Moore's law, the number of transistors that can be placed on an integrated circuit would approximately double every eighteen months.
}

separated by an insulating oxide, in such a way that the stack of the three materials forms a capacitor. A voltage applied to the gate is then used to control the resistance of the channel [4], and hence to amplify or switch electronic signals. Now, a large capacitance $C$ is mandatory in order to operate the transistor at low gate voltages. (The resistance of the channel depends on the "free charge" that is stored on the semiconductor side.)

Recalling the textbook formula for $C$ (the subscript "geom" emphasizes that, for a given dielectric material, its value only depends on the geometry of the parallel-plate capacitor),

$$
C=C_{\mathrm{geom}}=\frac{\kappa A}{4 \pi d},
$$

where $\kappa$ is the permittivity, $d$ is the thickness, and $A$ the surface area, one can see that miniaturization inevitably requires thinner dielectric layers if $C$ is to be kept constant upon a reduction of $A$.

Yet, when $d$ reaches the length scale of few (tens of) atomic layers, tunneling currents become so large that further shrinking would be impractical; this summarizes, in a nutshell, the conundrum that the semiconductor industry is currently facing.

To work around this issue, one would need to increase the capacitance per unit area without further reducing the dielectric thickness, a task in which both technology and fundamental research have invested tremendous efforts in the 
past few years. A particularly promising route revolves around the concept of "negative capacitance." Briefly, it consists in connecting two or more capacitors [e.g., the usual dielectric film described by Eq. (1), plus an additional element whose physical nature will be specified shortly] in series, in such a way that the overall capacitance is larger than the original value. Of course, if we stick to ordinary device elements, this is impossible: elementary electrostatics dictates that the total capacitance is always smaller than that of any individual capacitor in the series. However, if one of the capacitances is negative, then the total capacitance can, in principle, be larger than that of the constituents, i.e., $C$ could be enhanced with respect to the classically expected value, Eq. (1), without reducing the geometric thickness $d$.

The obvious question, then, is how to realize a negative capacitance in practice. Different proposals have appeared in the literature during the last few years. They can be more easily understood if we notice that the inverse of the capacitance can be written as the second derivative of the total energy with respect to the charge stored on the plates [5]. While a negative value may appear unphysical at first sight, as it indicates a thermodynamic instability of the system, a "negative capacitance" can nevertheless exist locally, in a composite device whose global capacitance is still positive.

A first possibility has already been demonstrated in ferroelectric nanocapacitors [6-9]. The basic idea is that a ferroelectric material has a switchable spontaneous polarization $(P)$, whose potential landscape $E(P)$ can be described by a characteristic double-well curve. In a vicinity of the centrosymmetric saddle-point configuration, such a curve is convex (i.e., $d^{2} E / d P^{2}<0$ ), indicating a polar instability; therefore, a ferroelectric film can in principle provide a negative contribution to $C$ when appropriately incorporated in a capacitor stack. ${ }^{2}$

A second strategy puts, instead, the emphasis on the physics of the metallic electrodes themselves. In particular, Kopp and Mannhart [10] recently argued that the total capacitance $C$ of a device depends on the quantum-mechanical nature of the electrodes via

$$
\frac{1}{C}=\frac{1}{C_{\text {geom }}}+\frac{1}{A e^{2}} \frac{d \mu}{d n} .
$$

Here, $C_{\text {geom }}$ is the classical capacitance of Eq. (1), $\mu$ is the chemical potential (Fermi level) of the metallic plate, $e$ is the electron charge, and $n$ is the electron density per surface area.

The second term on the right-hand side is proportional to the "electronic compressibility" $d \mu / d n$ and encodes the aforementioned electrode-dependent effects. Within specific conditions (dilute two-dimensional gases) that can, in principle, be realized in MOSFETs [11], an electron system can enter a regime of "negative electronic compressibility," i.e.,

\footnotetext{
${ }^{2}$ In a capacitor, the charge density stored on the plates is proportional to the normal component of the electric displacement field. Since, in atomic units, $Q / S=D /(4 \pi)=(E+4 \pi P) /(4 \pi)$, where $E$ is the normal component of the electric field, and in a typical ferroelectric material $4 \pi P \gg E$, then $Q / S \approx P$.
}

$d \mu / d n<0$, and hence provide a negative contribution to the overall capacitance.

Experimentally, this unconventional ${ }^{3}$ behavior was first detected in semiconductor (GaAs/AlGaAs) quantum wells $[12,13]$. The effect was ascribed to the quantum nature of the two-dimensional electrodes: in the dilute limit, the response of confined electron gases is typically dominated by exchange effects and tend to yield a negative $d \mu / d n$ [13]. The interest in this effect has remained mostly academic until recently because the relative gain in capacitance that one expects for semiconductor-based systems is too small for practical applications. However, fundamental research in this area has regained momentum with the recent discovery of a very large capacitance enhancements ( $>40 \%$ with respect to the geometrical value) in two-dimensional electron gas (2DEGs) in oxide nanostructures $\left(\mathrm{LaAlO}_{3} / \mathrm{SrTiO}_{3}\right.$ interface) $[14,15]$. On one hand, it is very tempting to interpret these arresting results as a manifestation of the same physics as that observed and modeled by Eisenstein et al. [13]. On the other hand, it is important to keep in mind that a polar oxide interface drastically departs, both from the point of view of structural and electronic properties, from the (much simpler) case of a semiconductor quantum well. In order to avoid any uncontrolled extrapolation it is, therefore, necessary to critically assess, first of all, the generality of the existing negative compressibility models, and verify whether their conclusions are general enough to encompass a wider range of materials and geometries.

In this work we quantitatively evaluate, based on firstprinciples calculations of jellium slabs and on analytic derivations, all the major ingredients that contribute to the electronic compressibility in ultrathin metallic electrodes. We devote special attention to the role played by electron confinement, which we identify as a key difference between the cases of the quasi-infinite square well (appropriate to deal with the case of a semiconductor quantum well, where the electrons are confined by large conduction band offsets), and that of the asymmetric wedgelike potentials (useful to describe polar interfaces). In the case of strong external confinement, our results show excellent agreement with both the model of Eisenstein et al. [13] and with the ideas that Kopp and Mannhart [10] brought forward to explain the origin of the negative capacitance in ideal two-dimensional systems. However, when the confining potential is modified (or removed altogether, leaving a jelliumlike positive background to keep the electrons in place) we find that the properties of the system are affected rather dramatically, in some cases even reversing the expected qualitative trends. First, we show that, in the weak confinement regime, the wave-function response to an external field is much stronger than in a quasi-twodimensional (2D) quantum well with essentially infinite potential barriers; this leads to a very strong charge-density contribution to the capacitance. This effect stems from the progressive displacement of the "image-charge" plane from

\footnotetext{
${ }^{3}$ In the standard textbook picture, when an electron is added to a metallic system it fills the lowest unoccupied energy state; as a consequence, the chemical potential increases.
} 
the geometric center of the 2D-like electrode while the capacitor is progressively charged [16], and always provides a negative contribution to $C$. (In fact, we find that this contribution is well appreciable even in the regime investigated in Ref. [13], although the experimental setup therein was not specifically designed to detect it.) Second, we find that a more delocalized nature of the electron gas facilitates population of higher subbands, and this introduces contributions to the capacitance that go well beyond the assumptions of Ref. [10]. In particular, when a new band starts to be populated we find a strong, abrupt drop in the electronic compressibility, which can become even more negative than that of an ideal 2D system at comparable density. This effect originates from the orthogonality between the wave functions that belong to different energy bands, which allows for a more efficient redistribution of the electron charge, and hence for a drastic reduction of the electrostatic (Hartree) contribution to the electronic compressibility.

Our work, therefore, highlights two previously overlooked mechanisms that can potentially lead to a negative contribution to the capacitance in interacting electron systems. We note both effects are completely general, and applicable to a wide variety of physical systems, as we illustrate via a variety of test cases. As a matter of fact, the "image-plane" chargedensity effect is not restricted to ultrathin quasi-2D metallic systems, but can be readily found in standard thick electrodes as well, where the concept of "negative compressibility" is not applicable. (The chemical potential is fixed by the Fermi level of the bulk metal.) At the same time, our work clearly shows that exceptional care is needed when dealing with two-dimensional electron systems: the underlying physical mechanisms at play may be substantially different from case to case, and critically depend on the structural and electronic properties of the host material.

Our work is organized as follows. In Sec. II we summarize the main features of our capacitor model. In Sec. II A we provide a brief background on the physics of the inverse capacitance density and its relation with the negative electron compressibility. We include the explicit formulation to compute it in the cases where only one band is occupied (Sec. II B), its relationship with perturbation theory (Sec. IIC), and the generalization to multiple occupied bands (Sec. IID). The self-consistent numerical implementation of all the above is described in Sec. IIE. The established theory of confined electron gases with frozen wave functions is revisited under the light of our prescription in Sec. III, including ideal twodimensional electron gases (Sec. III A) and the effects introduced by the finite thickness where the electron gas is confined (Sec. III B). In Sec. IV we demonstrate the importance of relaxing the wave functions in three different regimes: when the quantum gas is confined by a strong external potential, typical case in semiconductor quantum wells (QWs) (Sec. IV A), weak confinement (jellium slab in the absence of a strong confinement potential; Sec. IVC), and asymmetric barrier (reminiscent of a polar interface; Sec. IVD), highlighting in each case the connections to earlier works and the two original mechanisms that we introduced above. Finally, in Secs. V and VI we discuss some possible realizations of the ideas described here, together with some limitations of our treatment that may motivate further work on this subject.

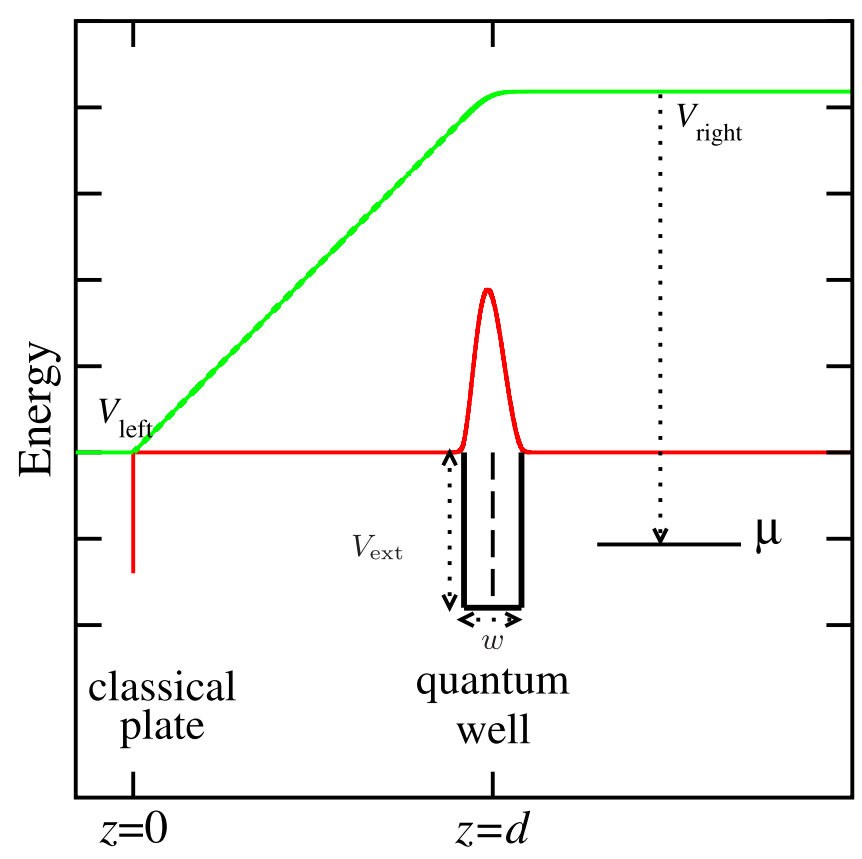

FIG. 1. Schematic view of the computational setup used in our model. The capacitor is made of a classical plate of zero thickness where a negative background charge is located (solid vertical red line at the left), and a quantum electrode, here represented as a quantum well of thickness $w$ where the electronic charge (solid red line) is localized. Green and black solid lines represent, respectively, the electrostatic potential and a strong external potential, typical in semiconductor QWs, that confine the quantum gas $\left(V_{\mathrm{ext}}\right)$. Vertical dashed line represents the geometrical center of the quantum well, whose distance with respect to the classical plate is $d$.

\section{METHOD}

In Fig. 1 we present a schematic version of the capacitor model that we shall use in this work. It consists in a classical electrode, whose surface is located at $z=0$, and of a quantum electrode, represented as a quantum well of thickness $w$ and centered at $z=d$; the two are separated by a dielectric of permittivity $\kappa$ [17]. In the remainder of this work, we shall assume that the system is infinitely extended in the $(x, y)$ plane, parallel to the electrode surface, and we shall indicate the perpendicular direction as $z$. Unless otherwise stated, we shall use atomic units throughout $\left(\hbar=m_{\mathrm{e}}=a_{0}=c=e=1\right)$. The electronic charge is assumed to be positive, so the electrostatic energy and the electrostatic potential, as well as the electron density and the electronic charge density, amount to the same value numerically. The in-plane electron density $n$ can also be described by the parameter $r_{\mathrm{s}}$, measured in Bohr units, which characterizes the average interparticle distance within the plane,

$$
\frac{1}{n}=\pi r_{\mathrm{s}}^{2}
$$

Within the quantum electrode, we shall explicitly solve either the many-body or mean-field Schrödinger equation, in order to account for all relevant electron-electron interactions at and beyond the Hartree description (further details of the 
numerical procedure are provided in Sec. II E). ${ }^{4}$ In particular, we shall write the total charge density as

$$
\rho_{\text {tot }}(z)=\left(n_{\text {jell }}-n\right) \delta(z)+\rho_{\text {el }}(z)+\rho_{\text {jell }}(z),
$$

where the delta function at $z=0$ describes the classical plate, $\rho_{\mathrm{el}}(z)$ is the electronic density of the quantum plate, and $\rho_{\text {jell }}(z)$ accounts for the possible inclusion of a uniform jellium background within the latter. The capacitor is overall neutral, so the integral of the total charge density written in Eq. (4) along the whole $z$ axis vanishes; this implies that $\int d z \rho_{\text {jell }}(z)=-n_{\text {jell }}$. The electrostatic potential $V_{\mathrm{H}}(z)$ is then calculated from $\rho_{\text {tot }}$ via a one-dimensional Poisson equation

$$
\frac{d^{2} V_{\mathrm{H}}(z)}{d z^{2}}=-\frac{4 \pi}{\kappa} \rho_{\mathrm{tot}}(z) .
$$

(We shall set the electrical boundary conditions in such a way that the electrostatic potential is constant and equal to $V_{\text {left }}$ for $z<0$. Due to the neutrality of the capacitor, the potential will be again constant for $z \gg d+w / 2$ and equal to $V_{\text {right }}$.)

Since $\rho_{\text {tot }}(z)$ differs from zero only in proximity of the capacitor plates, the electric field equals $\mathcal{E}=-d V_{\mathrm{H}}(z) / d z=$ $-4 \pi n / \kappa$ in the interior of the capacitor. We shall indicate the center of the charge density stored on the quantum electrode as the "average charge plane"

$$
\bar{z}=\frac{1}{n} \int d z z \rho_{\mathrm{tot}}(z)
$$

It is an easy exercise to verify that, in full generality and with the sign convention we have chosen,

$$
\frac{4 \pi n \bar{z}(n)}{\kappa}=V_{\mathrm{tot}}(n),
$$

where we have defined $V_{\text {tot }}=V_{\text {right }}-V_{\text {left }}$.

\section{A. Inverse capacitance density}

Knowledge of the electrostatic potential offset $V_{\text {tot }}$ is not enough to compute the inverse capacitance density $\mathcal{C}^{-1}=C^{-1} A$. The latter is defined as the first derivative of the Fermi level offset, or equivalently as the second derivative (with respect to $n$ ) of the total energy of the capacitor per surface unit $E_{\text {tot }}$ :

$$
\mathcal{C}^{-1}=\frac{d \mu_{\text {tot }}}{d n}=\frac{d^{2} E_{\text {tot }}}{d n^{2}} .
$$

Here, similarly to the potential, we have defined

$$
\mu_{\text {tot }}=\mu_{\text {right }}-\mu_{\text {left }}
$$

as the difference between the Fermi levels of the left $\left(\mu_{\text {left }}\right)$ and right $\left(\mu_{\text {right }}\right)$ electrodes. Given the assumption of an ideal classical electrode on the left, we can assume $\mu_{\text {left }}=V_{\text {left }}$; thus, $\mu_{\mathrm{tot}}$ is the Fermi level of the quantum electrode referred to $V_{\text {left }}$. Clearly, $\mu_{\text {tot }}$ is a geometry-dependent quantity: at a given $n$ the thicker the capacitor, the larger the potential

\footnotetext{
${ }^{4}$ Note that in Ref. [13] a slightly more complicated double-well setup was used, in order to facilitate the comparison with experimental measurements; the precise relationship between our model in Fig. 1 and that of Ref. [13] will be discussed in Sec. IV.
}

drop between the plates. To have a geometry-independent definition of the chemical potential it is convenient to use $V_{\text {right }}$ as a reference instead, and define

$$
\mu=\mu_{\text {right }}-V_{\text {right }} .
$$

Then, by construction, we have

$$
\mathcal{C}^{-1}=\frac{d V_{\text {tot }}}{d n}+\frac{d \mu}{d n}=\frac{4 \pi z_{\text {im }}}{\kappa}+\frac{d \mu}{d n},
$$

where we have introduced the differential center of charge $z_{\mathrm{im}}$, also known as "image-charge" plane,

$$
z_{\text {im }}(n)=\frac{d(n \bar{z})}{d n} .
$$

The relationship between $\bar{z}$ and $z_{\mathrm{im}}$ is in all respects analogous to that existing between average and instantaneous velocity, and reduces to an equality only in cases where the perturbation of the electronic wave function due to the applied bias can be neglected. By replacing Eq. (6) into Eq. (12), one can trivially verify that $z_{\mathrm{im}}$ is the first moment of charge-density variation with respect to $n$; in other words, it corresponds to the average location where the free electrons accumulate when an infinitesimal charge is added to the plate. $z_{\mathrm{im}}$ is thus uniquely given by the charge-density response of the system to an applied bias [recall Eq. (7)].

In Eq. (11) we have achieved an intuitive partition of $\mathcal{C}^{-1}$ into an "electrostatic" term which is reminiscent of the geometric contribution to the right-hand side of Eq. (2), and a "quantum" contribution, which depends on the Fermi level response of the electrode. Note, however, that the labels "electrostatic" and "quantum" should not be overinterpreted. On one hand, the electronic charge density response to a bias, which enters the definition of $z_{\mathrm{im}}$, is obviously determined by quantum effects; on the other hand, the evolution of the Fermi level contains residual electrostatic terms that are not accounted for by $z_{\mathrm{im}}$, as we shall see shortly. Thus, in the following we shall regard both as "quantum" contributions, and recover the "geometric" term of Eq. (2) by simply referring $z_{\text {im }}$ to some well-defined feature of the quantum electrode (e.g., the center of the well, as in the figure).

We stress that the choice of such reference is somewhat arbitrary: for example, there are equally good arguments to set $d$ either at the surface or at the center of the well; obviously, the concept of "geometric" distance between electrodes becomes ambiguous when the thickness of the device becomes comparable to the typical interatomic distances.

\section{B. Energy decomposition in a mean-field context}

In the remainder of this section we shall assume a singly occupied band, whose wave functions are given by the product of a plane wave in the plane of the quantum electrode (indexed by the in-plane wave number $\mathbf{k}_{\|}$) times an envelope function along $z$ :

$$
\psi_{\mathbf{k}_{\|}}(\mathbf{r})=e^{i \mathbf{k}_{\|} \cdot \mathbf{r}} \psi(z) .
$$

The one-dimensional envelope function $\psi(z)$ is normalized to unity, and corresponds to the self-consistent solution of the following eigenvalue problem:

$$
\hat{H}|\psi\rangle=\epsilon|\psi\rangle .
$$


Here, $\hat{H}=\hat{T}_{z}+\hat{V}_{\mathrm{H}}+\hat{V}_{\mathrm{xc}}+\hat{V}_{\mathrm{ext}}$ is the Kohn-Sham Hamiltonian. The first term on the right-hand side

$$
\hat{T}_{z}=-\frac{1}{2 m_{\perp}} \frac{d^{2}}{d z^{2}}
$$

is the normal component of the kinetic energy operator, where $m_{\perp}$ is the effective mass along $z$.

The other terms are the Hartree $(\mathrm{H})$, exchange-correlation (xc), and external (ext) potentials. The Hartree and exchangecorrelation potentials, in turn, depend on the total and electronic charge densities, where the latter is defined in term of the wave function $\psi(z)$ as

$$
\rho_{\mathrm{el}}(z)=n|\psi(z)|^{2} .
$$

The total energy of the capacitor can then be written as a variational functional of $\psi(z)$ that depends parametrically on the areal density of particles $n$, as

$$
E_{\mathrm{tot}}(\psi, n)=E_{\mathrm{K}}+E_{\mathrm{H}}+E_{\mathrm{xc}}+E_{\mathrm{ext}}-\lambda(\langle\psi \mid \psi\rangle-1) .
$$

Here,

$$
E_{\mathrm{K}}=\frac{\pi n^{2}}{2 m_{\|}}+n\left\langle\psi\left|\hat{T}_{z}\right| \psi\right\rangle
$$

is the kinetic energy density of the noninteracting electrons, which contains the trivial in-plane contribution (depending quadratically on $n$ and inversely on the in-plane effective mass $\left.m_{\|}\right)$

$$
E_{\mathrm{H}}\left[\rho_{\mathrm{tot}}\right]=\frac{1}{2} \int d z V_{\mathrm{H}}(z) \rho_{\mathrm{tot}}(z)
$$

is the electrostatic energy density, where the Hartree potential is defined by Eq. (5):

$$
E_{\mathrm{xc}}\left[\rho_{\mathrm{el}}\right]=\int d z \epsilon_{\mathrm{xc}}\left[\rho_{\mathrm{el}}(z)\right] \rho_{\mathrm{el}}(z)
$$

is the exchange and correlation energy density (describing the electron-electron interactions beyond the Hartree approximation, plus the remainder of the many-body kinetic energy). Finally,

$$
E_{\mathrm{ext}}\left[\rho_{\mathrm{el}}\right]=\int d z V_{\mathrm{ext}}(z) \rho_{\mathrm{el}}(z)
$$

accounts for a possible external potential. The last term in Eq. (17) is a Lagrange multiplier, and serves to guaranteeing the correct normalization of the wave function; at the variational minimum one has $\lambda=n \epsilon$.

We shall now assume that we are at the variational minimum with respect to $\psi$, i.e., define

$$
E_{\text {tot }}(n)=\min _{\psi} E_{\text {tot }}(\psi, n) .
$$

To connect the present treatment with the quantities that we introduced in the earlier Sec. II A, we shall then take the total derivative of $E_{\text {tot }}(n)$ with respect to $n$. The latter, in virtue of the Hellmann-Feynman theorem, reduces to a partial derivative and can be easily calculated:

$$
\frac{d E_{\mathrm{tot}}}{d n}=\frac{\partial E_{\mathrm{tot}}}{\partial n}=\frac{\pi n}{m_{\|}}+\epsilon-V_{\mathrm{left}} .
$$

[Note that the eigenvalue $\epsilon$ and $V_{\text {left }}$ are both defined modulo an arbitrary constant, which stems from the electrostatic potential $V_{\mathrm{H}}(z)$ as given by Poisson's equation (5); such arbitrariness cancels out when taking their difference.] To arrive at this result, we have used the following relationship between self-consistent potentials and energies:

$$
\frac{\delta E_{\mathrm{H}, \mathrm{xc}}[\rho]}{\delta \rho(z)}=V_{\mathrm{H}, \mathrm{xc}}(z)
$$

and we have subsequently used Eq. (14) to replace $\langle\psi|\hat{H}| \psi\rangle=\epsilon$. Consistent with Eq. (8), it is easy to get convinced that Eq. (23) describes the chemical potential of the quantum electrode, with $V_{\text {left }}$ used as a reference, i.e., $\mu_{\text {tot }}$.

To make progress toward an expression for $\mathcal{C}^{-1}$, one can again use the Hellmann-Feynman theorem to write

$$
\begin{aligned}
\mathcal{C}^{-1}=\frac{d \mu_{\mathrm{tot}}}{d n} & =\frac{\pi}{m_{\|}}+\frac{d\left(\epsilon-V_{\text {left }}\right)}{d n} \\
& =\frac{\pi}{m_{\|}}+\left\langle\psi\left|\frac{d \hat{H}}{d n}\right| \psi\right\rangle-\frac{d V_{\text {left }}}{d n} .
\end{aligned}
$$

Note the total derivative signs, which imply that the variation of the self-consistent potentials with $n$, due to the relaxation of the wave functions $d|\psi\rangle / d n$, must be included.

In fact, since the Hamiltonian only depends on $n$ via the Hartree and exchange-correlation potentials, we have

$$
\mathcal{C}^{-1}=\frac{\pi}{m_{\|}}+\Delta_{\mathrm{H}}^{\text {left }}+\Delta_{\mathrm{xc}},
$$

where the first term on the right-hand side is the constant kinetic contribution, related to the in-plane dispersion, and the other two terms refer to the Hartree $(\mathrm{H})$ and exchangecorrelation (xc) contributions to the eigenvalue variation

$$
\begin{aligned}
\Delta_{\mathrm{H}}^{\mathrm{left}} & =\left\langle\psi\left|\frac{d\left(\hat{V}_{\mathrm{H}}-V_{\text {left }}\right)}{d n}\right| \psi\right\rangle, \\
\Delta_{\mathrm{xc}} & =\left\langle\psi\left|\frac{d \hat{V}_{\mathrm{xc}}}{d n}\right| \psi\right\rangle .
\end{aligned}
$$

To complete the link to the results of the previous section, note that in Eq. (26) we have incorporated the variation of $V_{\text {left }}$ into the Hartree contribution. This has been done on purpose: Indeed, subtracting $V_{\text {left }}$ from $V_{\mathrm{H}}(z)$ boils down, from the physical point of view, to fixing the reference of the electrostatic potential. The drawback of such a choice is that $\Delta_{\mathrm{H}}^{\text {left }}$ depends on the geometry of the capacitor, i.e., on the distance between the plates.

To circumvent this issue, we can proceed in pretty much the same way as before, by setting the convention to $V_{\text {right }}=0$ instead,

$$
\Delta_{\mathrm{H}}^{\text {left }}=\Delta_{\mathrm{H}}^{\text {right }}+\frac{4 \pi z_{\mathrm{im}}}{\kappa},
$$

where

$$
\Delta_{\mathrm{H}}^{\text {right }}=\left\langle\psi\left|\frac{d\left(\hat{V}_{\mathrm{H}}-V_{\text {right }}\right)}{d n}\right| \psi\right\rangle .
$$

Then, $\Delta_{\mathrm{H}}^{\text {right }}$ reflects the variation of the internal electrostatic energy of the "quantum" plate (we shall see that this term can be identified with the Hartree band-bending effect), while the 
second one is the familiar image-plane contribution. Summarizing all the previous results in a single expression, in the spirit of Eq. (11),

$$
\begin{aligned}
\mathcal{C}^{-1} & =\frac{4 \pi z_{\mathrm{im}}}{\kappa}+\frac{d \mu}{d n} \\
& =\frac{4 \pi z_{\mathrm{im}}}{\kappa}+\frac{\pi}{m_{\|}}+\Delta_{\mathrm{H}}^{\text {right }}+\Delta_{\mathrm{xc}} .
\end{aligned}
$$

Similarly, the Fermi level referred to $V_{\text {right }}$ is

$$
\mu=\frac{\pi n}{m_{\|}}+\epsilon-V_{\text {right }} \text {. }
$$

\section{Relationship to perturbation theory}

With the above derivations, we have achieved a further insight into the physics of the inverse capacitance density, by separating it into a trivial (constant) kinetic term and two contributions (Hartree and $\mathrm{xc}$ ) that originate from the variation of eigenvalue $\epsilon$ with $n$.

Before moving on, it is worth spending a few words on the specific role played by the relaxation of the electronic wave function since its effect is not immediately clear from the above derivations. To see this, it is useful to reformulate the inverse capacitance problem in the language of linearresponse theory [18].

In such a framework, the energy functional of Eqs. (17) and (22) can be expanded to second order in the perturbation parameter $n$ around some reference value $n_{0}$ [we shall indicate the $l$ th total derivative with respect to $n$ with a $(l)$ superscript henceforth],

$$
\begin{aligned}
E_{\mathrm{tot}}(n)= & E_{\mathrm{tot}}\left(n_{0}\right)+\left(n-n_{0}\right) E^{(1)}\left(n_{0}\right) \\
& +\frac{\left(n-n_{0}\right)^{2}}{2} E^{(2)}\left(n_{0}\right)+\cdots,
\end{aligned}
$$

where the first- and second-order terms are, respectively, the chemical potential and the inverse capacitance density,

$$
E^{(1)}=\mu_{\mathrm{tot}}, \quad E^{(2)}=\mathcal{C}^{-1} .
$$

An analogous expansion can be operated on the wave functions

$$
\psi(n)=\psi\left(n_{0}\right)+\left(n-n_{0}\right) \psi^{(1)}\left(n_{0}\right)+\cdots .
$$

One can then write explicit expressions for the $(2 j+1)$ th term in the expansion of the energy functionals by using the expansion terms of $\psi$ up to order $j$.

Crucially, the even-order ( $2 j$ th) energy expansion terms can be constructed in such a way that they are variational in the $j$ th-order wave functions [19]; this implies that the inverse capacitance density can be written as a variational functional of the first-order wave functions $\psi^{(1)}$ :

$$
\begin{aligned}
E^{(2)}\left(\psi^{(1)}, n\right)= & \frac{\pi}{m_{\|}}+2 n\left\langle\psi^{(1)}|(H-\epsilon)| \psi^{(1)}\right\rangle \\
& +\rho_{\mathrm{tot}}^{(1)} \cdot K_{\mathrm{H}} \cdot \rho_{\mathrm{tot}}^{(1)}+\rho_{\mathrm{el}}^{(1)} \cdot K_{\mathrm{xc}} \cdot \rho_{\mathrm{el}}^{(1)} .
\end{aligned}
$$

Here, $K_{\mathrm{H}, \mathrm{xc}}$ indicates the Hartree or xc kernels,

$$
K_{\mathrm{H}, \mathrm{xc}}\left(z, z^{\prime}\right)=\frac{\delta^{2} E_{\mathrm{H}, \mathrm{xc}}}{\delta \rho(z) \delta \rho\left(z^{\prime}\right)},
$$

and the two first-order densities refer to the first-order variation of either the total or electronic charge density with $n$ :

$$
\begin{aligned}
& \rho_{\mathrm{el}}^{(1)}(z)=|\psi(z)|^{2}+2 n \psi(z) \psi^{(1)}(z), \\
& \rho_{\mathrm{tot}}^{(1)}(z)=\rho_{\mathrm{el}}^{(1)}(z)-\delta(z) .
\end{aligned}
$$

Such densities, in turn, define the first-order potentials via

$$
\begin{aligned}
\frac{d^{2} V_{\mathrm{H}}^{(1)}(z)}{d z^{2}} & =-\frac{4 \pi}{\kappa} \rho_{\mathrm{tot}}^{(1)}(z), \\
V_{\mathrm{xc}}^{(1)}(z) & =K_{\mathrm{xc}} \cdot \rho_{\mathrm{el}}^{(1)}(z) .
\end{aligned}
$$

It is straightforward to verify that Eq. (36) reduces to Eq. (26) at the variational minimum; in the language of Ref. [20] these two formulas can be regarded, respectively, as the stationary and nonstationary expressions for the inverse capacitance density.

In our context, the variational character of Eq. (36) implies that the relaxation of the wave functions always lowers the inverse capacitance density, whatever is the relative contribution of the individual effects. (Note that this statement is true for the total inverse capacitance density, while it may break down once we separate it into a "quantum" and "image-plane" contribution.) We shall illustrate this important point with our numerical experiments shortly.

\section{Multiband case}

So far we have discussed the inverse capacitance density in the special case of a single occupied band. We shall now extend the theory to the case of multiple bands. Assume that we have $N$ partially occupied bands of the form

$$
\psi_{\mathbf{k}_{\|}, l}(\mathbf{r})=e^{i \mathbf{k}_{\|} \cdot \mathbf{r}} \psi_{l}(z) .
$$

Note that the individual $\psi_{l}$ need not be eigenstates of the same one-dimensional Schrödinger equation; in the most general case (as it happens, for instance, at the $\mathrm{LaAlO}_{3} / \mathrm{SrTiO}_{3}$ interface) [21], different types of subbands may be populated, where each subset feels a different external potential, and has a different out-of-plane dispersion $m_{\perp}(l)$. We shall further assume, for the sake of generality, that the in-plane effective mass of each state is $m_{\|}(l)$ (i.e., they need not be the same for all bands). Each band then contributes to the electronic charge density as

$$
\rho_{\mathrm{el}}(z)=\sum_{l} n_{l}\left|\psi_{l}(z)\right|^{2}
$$

with

$$
n_{l}=\frac{k_{\mathrm{F}}^{2}(l)}{2 \pi}, \quad \sum_{l} n_{l}=n,
$$

where $k_{\mathrm{F}}^{2}(l)$ is the Fermi momentum, and to the total kinetic energy as

$$
E_{\mathrm{K}}(l)=\frac{\pi n_{l}^{2}}{2 m_{\|}(l)}, \quad \sum_{l} E_{\mathrm{K}}(l)=E_{\mathrm{K}} .
$$

Finally, since there is a unique Fermi level, the quantity

$$
\mu_{\mathrm{tot}}=\frac{\pi n_{l}}{m_{\|}(l)}+\epsilon_{l}-V_{\mathrm{left}}
$$


must be the same for all bands. Solving for $n_{l}$ in Eq. (46) and replacing in Eq. (44), we obtain

$$
\frac{\sum_{l} m_{\|}(l)}{\pi} \mu_{\mathrm{tot}}=n+\sum_{l} \frac{m_{\|}(l)}{\pi}\left(\epsilon_{l}-V_{\mathrm{left}}\right)
$$

and finally

$$
\mu_{\mathrm{tot}}=\frac{\pi n}{\sum_{l} m_{\|}(l)}+\frac{\sum_{l} m_{\|}(l) \epsilon_{l}}{\sum_{l} m_{\|}(l)}-V_{\mathrm{left}}
$$

which yields the chemical potential as a function of the total charge density $n$ and of a weighted sum of the single-particle eigenvalues.

As it was done in Sec. II B for the single-band case, the inverse capacitance density can be computed as

$$
\mathcal{C}^{-1}=\frac{d \mu_{\mathrm{tot}}}{d n}=\frac{\pi}{\sum_{l} m_{\|}(l)}+\frac{\sum_{l} m_{\|}(l) \frac{d \epsilon_{l}}{d n}}{\sum_{l} m_{\|}(l)}-\frac{d V_{\text {left }}}{d n} .
$$

The most remarkable consequence of Eq. (49) is a drastic lowering of the positive contribution to the compressibility that is due to the in-plane kinetic energy. Indeed, if we assume that the masses are all equal, the kinetic contribution to $\mathcal{C}^{-1}$ is $\pi /\left(N m_{\|}\right)$, i.e., it reduces to a fraction of the single-band value $\pi / m_{\|}$. Regarding the eigenvalue contribution, it is straightforward to show that

$$
\frac{d \epsilon_{l}}{d n}=\left\langle\psi_{l}\left|\left(\hat{V}_{\mathrm{H}}^{(1)}+\hat{V}_{\mathrm{xc}}^{(1)}\right)\right| \psi_{l}\right\rangle,
$$

i.e., even if the states $\psi_{l}$ are eigenfunctions of different Hamiltonian operators, only the variation of the global selfconsistent potential is relevant for calculating their contribution to $\mathcal{C}^{-1}$.

\section{E. Computational method}

We have implemented the model presented in the previous sections by self-consistently solving the one-particle KohnSham Hamiltonian described in Eq. (14). To perform the integrals, we use Numerov's algorithm on a real-space grid, whose fineness can be controlled with a single energy cutoff (the kinetic energy of the plane wave that can be represented in the grid without aliasing).

The exchange and correlation interactions are treated at the level of the local density approximation (LDA) [22,23] to density functional theory (DFT) [24]. The energy eigenvalues of Eq. (14) corresponding to eigenfunctions with the correct asymptotic behavior (smooth decay outside the slab) are searched using the "shooting method" and the "double integration technique" [25]. Within this method, the eigenvalues of the bound states are always bounded between the minimum value of the total potential inside the supercell and the energy at left of the classical plate, taken as zero. At odds with other techniques that rely on the solution of the Poisson equation [Eq. (5)] with fast-Fourier transform techniques, where the eigenvalues are computed with respect to the average of the electrostatic potential in the unit cell (not always a welldefined quantity [26]) our reference energy is well defined and the eigenvalues between different calculations perfectly comparable.

\section{ELECTRON COMPRESSIBILITY IN CONFINED METALLIC GASES WITH FROZEN WAVE FUNCTIONS}

It is clear from Eq. (26) for the single-band case, or its generalization to the multiple-band case in Eqs. (49) and (50), that there are three main ingredients to the inverse capacitance density of an electron gas: The first one is a positive constant, independent of the electron density, that comes from the contribution of the in-plane kinetic energy. The second and the third are, respectively, the Hartree and the exchangecorrelation contributions to the eigenvalue variations. These three contributions have already been discussed at length in the context of confined electron gases. To prepare for the discussion of these effects, in this section we shall briefly review the established results, and link to the relevant literature whenever possible.

\section{A. Ideal two-dimensional case}

Pioneer works on negative capacitance relied on an ideal two-dimensional electron gas model (the electrons are exactly confined into an idealized 2D plane, with a homogeneous charge density) to rationalize the experimental results. In such a limit, the subbands are separated by an infinite energy so only the lowest one is occupied, and the electronic charge density has a shape of a Dirac delta located at $z=d$, i.e., at the distance between the classical electrode and the ideal plane.

In the limit of a two-dimensional electrode, the exchange energy per unit area $E_{\mathrm{x}}$ is negative and depends on the charge density as

$$
E_{\mathrm{x}}=\epsilon_{\mathrm{x}}^{2 \mathrm{D}}(n) n,
$$

where $\epsilon_{\mathrm{x}}^{2 \mathrm{D}}(n)$ is the exchange energy per electron

$$
\epsilon_{\mathrm{x}}^{2 \mathrm{D}}(n)=-\frac{4}{3 \kappa} \sqrt{\frac{2}{\pi}} n^{1 / 2} .
$$

Specializing the definition of the potential (24) to the exchange energy, then Eqs. (27) and (28) take the form

$$
\begin{aligned}
\Delta_{\mathrm{H}}^{\text {left }} & =\int d z V_{\mathrm{H}}^{(1)}(z)[\delta(z-d)-\delta(z)] \\
& =\frac{d V_{\text {right }}}{d n}-\frac{d V_{\text {left }}}{d n}=\frac{4 \pi d}{\kappa}, \\
\Delta_{\mathrm{xc}} & =\int d z V_{\mathrm{xc}}^{(1)}(z) \delta(z-d)=-\frac{1}{\kappa} \sqrt{\frac{2}{\pi}} n^{-1 / 2},
\end{aligned}
$$

where we have used that in this ideal two-dimensional case $z_{\mathrm{im}}=d$. Thus, following Eq. (26), the inverse of the capacitance density amounts to

$$
\mathcal{C}^{-1}=\frac{4 \pi d}{\kappa}+\left(\frac{\pi}{m_{\|}}-\frac{1}{\kappa} \sqrt{\frac{2}{\pi}} n^{-1 / 2}\right) .
$$

We can identify the quantity in brackets with the electronic compressibility $d \mu / d n$.

Its behavior as a function of $n$ is illustrated in Fig. 2. We can see that in the high-density limit (small $r_{\mathrm{s}}$ ) the electronic compressibility is positive and approaches the noninteracting regime, where the kinetic energy dominates. As the density is reduced, $d \mu / d n$ decreases, and within the present HartreeFock approximation, changes its sign at a critical density 


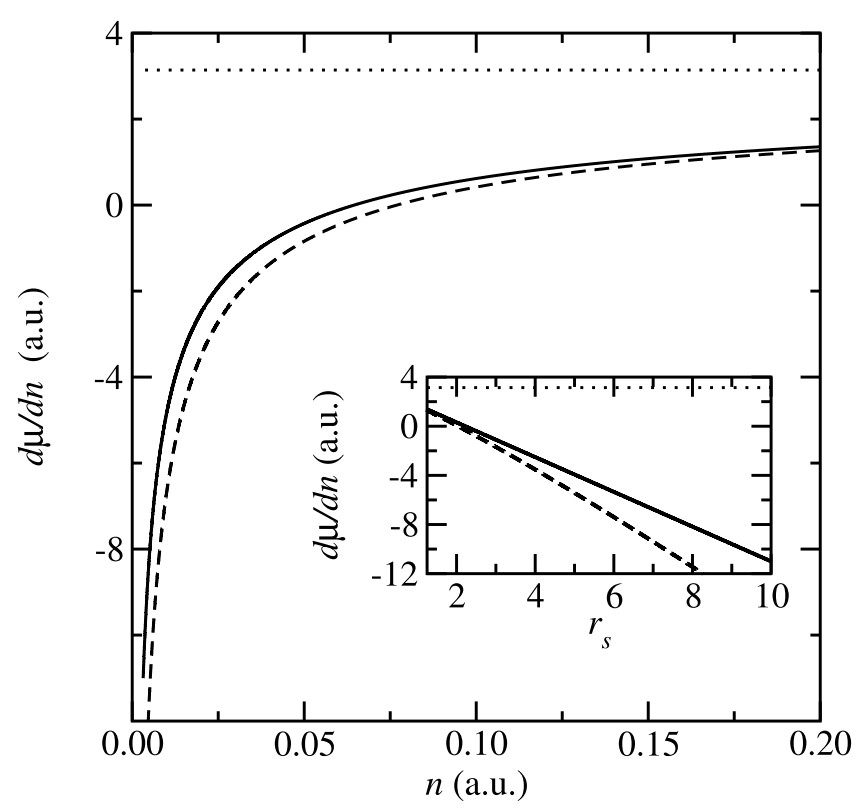

FIG. 2. Contributions to the electronic compressibility as a function of the charge density of the sheet in the ideal 2D limit. Black dotted line shows the constant kinetic contribution. Solid black line is the electronic compressibility (sum of the kinetic and exchange contribution). Dashed black curve considers, in addition, the correlation contribution. Inset: same results with respect the parameter $r_{\mathrm{s}}$. Results have been obtained for $m_{\|}=\kappa=1$.

$n_{c}=2 m_{\|}^{2} /\left(\kappa^{2} \pi^{3}\right)$. For lower densities, the electronic compressibility is negative and, therefore, as highlighted in the Introduction, the total capacitance can become larger than the classical geometrical value.

So far, we have neglected the correlation energy. Indeed, its effects [27] are expected to be minor within the density range of interest for typical semiconductors [13]. To assess the importance of correlation in the present context, we have recalculated $\mathcal{C}^{-1}$ by incorporating it explicitly (see dashed line in Fig. 2); its impact is marginal, as expected.

\section{B. Confined electron gas of a finite thickness}

In most practical cases, a confined electron gas significantly deviates from the ideal 2D model of Sec. III A because of finite-thickness effects [13]. In the following we shall recap the impact of Coulomb softening, affecting the mutual repulsion of the electrons, and of the Hartree band bending, i.e., a Stark-type shift of the quantum well states that is due to the external potential.

\section{Coulomb softening}

Coulomb softening has to do with the modification of the exchange interactions within the "thickened" gas. To gauge its importance, we shall assume that the gas is confined by an infinite square well of thickness $w$. This is justified in semiconductor quantum wells, whenever the conduction band offsets are much deeper than the mean electron energy. The electronic ground-state wave function can be then determined

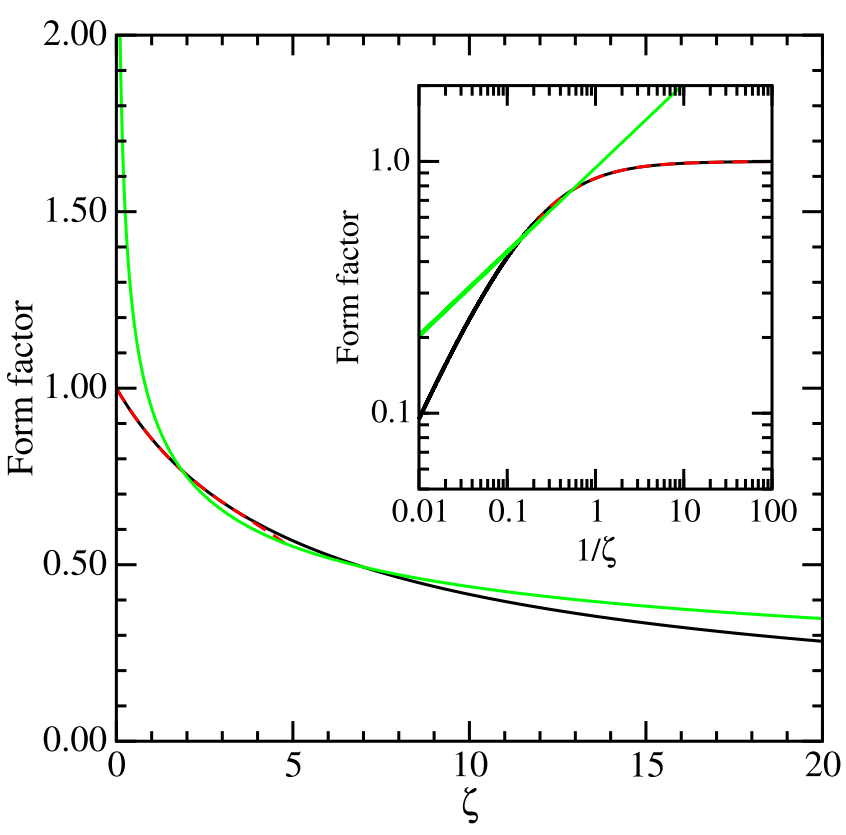

FIG. 3. Form factor as given by the exact exchange functional (black solid line), by a polynomial fit to low values of $\zeta=w / r_{\mathrm{s}}$ as fitted by Eisenstein et al. in Ref. [13] (red dashed lines), and by the LDA approximation (solid green line). Inset: same data, but in a log$\log$ scale and with the abscissas inverted.

analytically,

$$
\psi(z)=\sqrt{\frac{2}{w}} \sin \left(\frac{\pi z}{w}\right),
$$

leading to a three-dimensional charge density of the form

$$
\rho_{\mathrm{el}}(n, z)=n|\psi(z)|^{2}=\frac{2 n}{w} \sin ^{2}\left(\frac{\pi z}{w}\right) .
$$

Assuming that only the lowest subband is occupied within the relevant range of $n$, the necessary corrections to the exchange energy per electron, Eq. (52), can be summarized [28] in a form factor $F(\zeta)$,

$$
\epsilon_{\mathrm{x}}(n, w)=\epsilon_{\mathrm{x}}^{2 \mathrm{D}}(n) F(\zeta)
$$

where $\zeta=w / r_{\mathrm{s}}=w \sqrt{\pi n}$ is the dimensionless ratio between the width of the potential well and the mean spacing (in units of Bohr radius $a_{0}$ ) between electrons in the plane, as defined in Eq. (3). A simple polynomial fit, valid for an infinite square well ground-state wave function and for $0<\zeta<3.5$, is reported in the Appendix of Ref. [13]. Here, in order to obtain a more complete picture, we have calculated numerically $F(\zeta)$ over a broader range of densities/thicknesses. The results are shown in Fig. 3. Clearly, the exchange energy is always smaller than in the ideal 2D case (the form factor, black line in Fig. 3 is smaller than 1 at any $n$ ).

Many practical implementations, including the numerical model that we shall use in this work, rely on the use of approximate functionals for the exchange and correlation, e.g., the LDA [22,23]. It is therefore important, at this stage, to gauge the accuracy of the LDA exchange in describing the compressibility of a confined electron gas. In fact, just like in the case of the exact (Hartree-Fock) treatment, we can write 

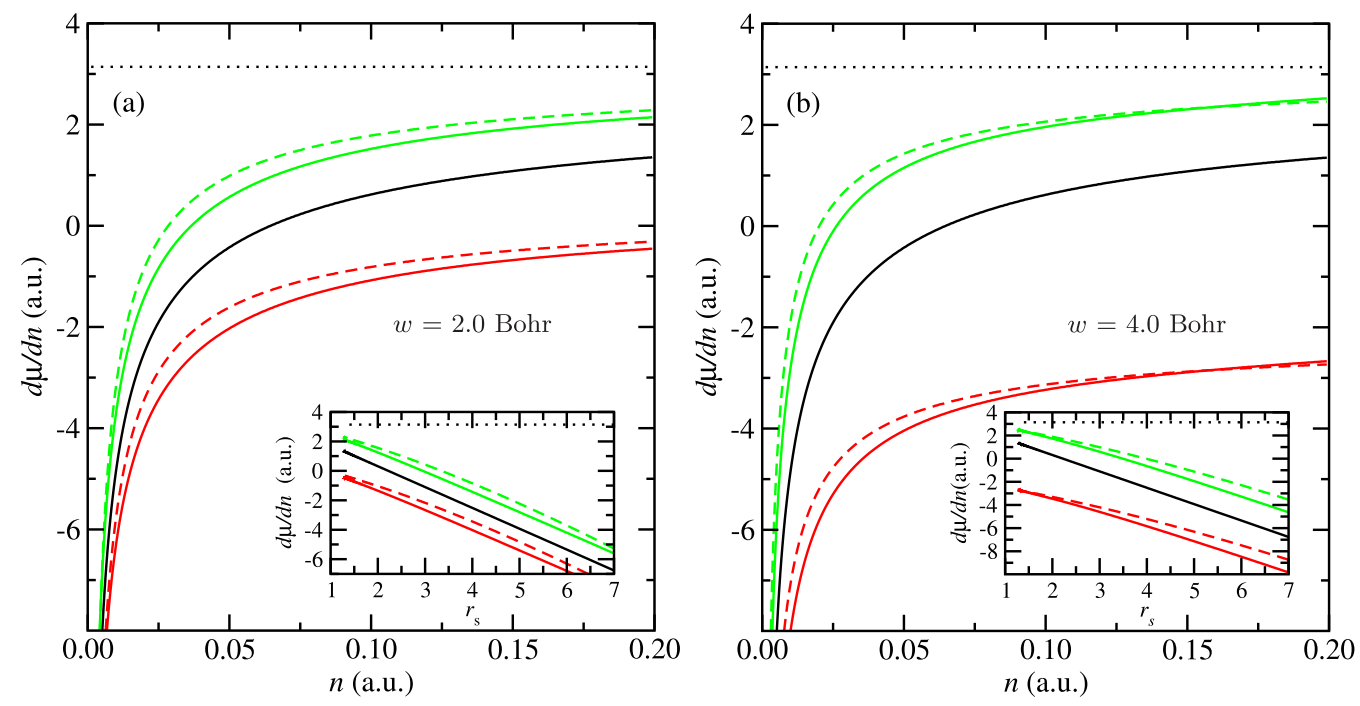

FIG. 4. Various contributions to the electronic compressibility as a function of the electron density, as given by the LDA exchange (dashed curves) or by the "thickened" (exact) exchange functional (solid curves). Results in (a) and (b) have been obtained for a thickness of $w=2.0$ and 4.0 bohrs, respectively. The analytical result for the ideal 2D limit ( $w \rightarrow 0$ limit) is also shown as a thick black curve for comparison. The dotted line at $d \mu / d n=\pi$ shows the contribution of the in-plane kinetic energy. Green curves represent the results considering only the thickened exchange, as discussed in Sec. III B 1, in addition to the noninteracting constant coming from the kinetic contribution. Red curves contain also the "Hartree band bending" as explained in Sec. III B 2. Insets: same results with respect to the parameter $r_{\mathrm{s}}$. Results have been obtained for $m_{\|}=\kappa=1$. a.u. stands for atomic units.

$\epsilon_{\mathrm{x}}^{\mathrm{LDA}}(n, w)$ as the ideal $2 \mathrm{D}$ exchange energy times a form factor

$$
\epsilon_{\mathrm{x}}^{\mathrm{LDA}}(n, w)=\epsilon_{\mathrm{x}}^{2 \mathrm{D}}(n) F^{\mathrm{LDA}}(\zeta),
$$

whose explicit form is

$$
F^{\mathrm{LDA}}(\zeta)=A \zeta^{-\frac{1}{3}}
$$

( $A=0.9436555$ is a dimensionless constant. $)$ The derivation of Eq. (60) is given in Appendix A.

The approximate LDA form factor is compared with the exact Hartree-Fock limit in Fig. 3. While the two functions roughly agree for $1<\zeta<10$, the LDA result is clearly wrong both in the low-density and high-density limits for a given $w$ (or, equivalently, in the large and small thickness limit for a given $n$.) In either case, LDA overestimates the exchange energy, and such an overestimation becomes severe at small $\zeta$ where $F^{\mathrm{LDA}}$ erroneously diverges as $\zeta^{-\frac{1}{3}}$. (This is the limit of small $w$ for a fixed $n$, where the exact result tends to 1.) These results indicate that outside the range $1<\zeta<10$, LDA may give a very inaccurate description of exchange effects, and one should be extremely careful when drawing physical conclusions therein.

Results including the contribution of this "Coulombsoftening" effect on the electronic compressibility are shown with green lines in Fig. 4. To quantify this softening, we use both the form factor computed numerically under the assumption of a frozen wave function corresponding to the ground state of an infinite square well potential (shown in Fig. 3), and the LDA form factor [Eq. (60)]. Then, the exchange energy per unit area is computed as in Eq. (51), together with its second derivative with respect to the charge density to evaluate numerically the contribution of the thickened exchange [Eq. (28)] to the electronic compressibility. After adding the constant contribution coming from the kinetic energy term, the most important conclusions that can be drawn are (i) due to the softening of the negative contributions coming from the exchange energy, this effect reduces the tendency of the electronic compressibility toward negative values (green curves in Fig. 4 are always above the analytical results for the ideal 2D limit, represented by the black curves); and (ii) in the ranges of densities and thicknesses tried in Fig. 4, the LDA approximation provides a qualitative (even semiquantitatively) correct picture when compared with the exact functional.

\section{Hartree band bending}

The Hartree band-bending effect is embodied in the $\Delta_{\mathrm{H}}^{\text {right }}$ contribution to the inverse capacitance density of Eq. (31), and is related to the first-order variation of the Hartree potential with $n$. At a given $n$, the Hartree potential is given by the Poisson equation of Eq. (5), which results in the following double integral:

$$
\begin{aligned}
V_{\mathrm{H}}(z) & =-\frac{4 \pi}{\kappa} \int_{-\infty}^{z} d t \int_{-\infty}^{t} d t^{\prime} \rho_{\mathrm{tot}}\left(t^{\prime}\right) \\
& =\frac{4 \pi n z}{\kappa}-\frac{4 \pi}{\kappa} \int_{-\infty}^{z} d t \int_{-\infty}^{t} d t^{\prime} n\left|\psi\left(t^{\prime}\right)\right|^{2} .
\end{aligned}
$$

Assuming that the wave function is frozen, then $V_{\mathrm{H}}(z)$ is linear in $n$, so its variation can be trivially computed:

$$
\frac{\delta V_{\mathrm{H}}(z)}{\delta n}=\frac{4 \pi z}{\kappa}-\frac{4 \pi}{\kappa} \int_{-\infty}^{z} d t \int_{-\infty}^{t} d t^{\prime}\left|\psi\left(t^{\prime}\right)\right|^{2}
$$


The integrals of Eqs. (27) and (62) can be calculated numerically, resulting in

$$
\Delta_{\mathrm{H}}^{\text {left }}=\frac{4 \pi \bar{z}}{\kappa}-1.29862 w,
$$

i.e., this is a constant contribution that simply scales linearly with the thickness of the quantum well. Since we have assumed that the electronic wave functions are frozen, $z_{\mathrm{im}}=$ $\bar{z}=d$ as explained in Sec. II A. Therefore, the first term of the right-hand side of Eq. (63) is the geometric contribution to the inverse of the capacitance density, while the remainder is the desired Hartree band-bending term $\Delta_{\mathrm{H}}^{\text {right }}=-1.29862 \mathrm{w}$.

Numerical results including the contribution of $\Delta_{\mathrm{H}}^{\text {right }}$ are shown in Fig. 4. Consistent with earlier studies, the (negative) Hartree band bending clearly overcompensates the Coulomb softening, resulting in an overall enhancement of the negative compressibility effect (red curves in Fig. 4 are always lower than the black ones). The thicker the film, the larger the reduction in the electron compressibility.

\section{Quantum wells}

The above arguments are valid for an electron gas confined in a potential well of infinite depth. To prepare for the discussion of our numerical results in the next section, we shall briefly study here the more realistic case of a quantum well (QW) with finite depth. This will allow us to gain a first insight on the impact of confinement effects.

To do so, we first calculate the finite-QW ground-state wave function of the bare well $(n=0)$ for three different depths $\left(V_{\text {ext }}=\infty, 250 \mathrm{Ha}\right.$, and $\left.22 \mathrm{Ha}\right)$. Next, we insert this wave function into Eq. (62) to compute the Hartree band bending at different values of $n$, neglecting relaxation as before. The results are plotted in Fig. 5. Almost no difference is found between the infinite quantum well and the 250-Ha one. Nevertheless, the shallower the quantum well, the more extended the wave functions, which results in a lowering of the electronic compressibility as if the QW were still infinite but slightly wider [the prefactor in front of the second term of the right-hand side of Eq. (63) changes to -1.351 for a depth of $250 \mathrm{Ha}$, and to -1.495 for a depth of $22 \mathrm{Ha}$ ].

Note that the Coulomb softening effect appears to be much less sensitive to the well depth compared to the Hartree bandbending term shown in Fig. 5. This observation is general to all our work. Indeed, we shall see in the following section that it is the Hartree band-bending effect that undergoes the most dramatic enhancements when the confinement is reduced or lifted altogether.

\section{ELECTRON COMPRESSIBILITY IN CONFINED METALLIC GASES WITH SELF-CONSISTENT WAVE FUNCTIONS}

After reviewing the basic phenomenology of confined 2D electrodes, we shall now move to presenting our main results, obtained by using the self-consistent numerical solver described in Sec. IIE. In particular, we shall focus on two distinct aspects of the problem: (i) the self-consistent relaxation of the wave functions, which leads to a displacement of the electronic charge density from the geometric center of the

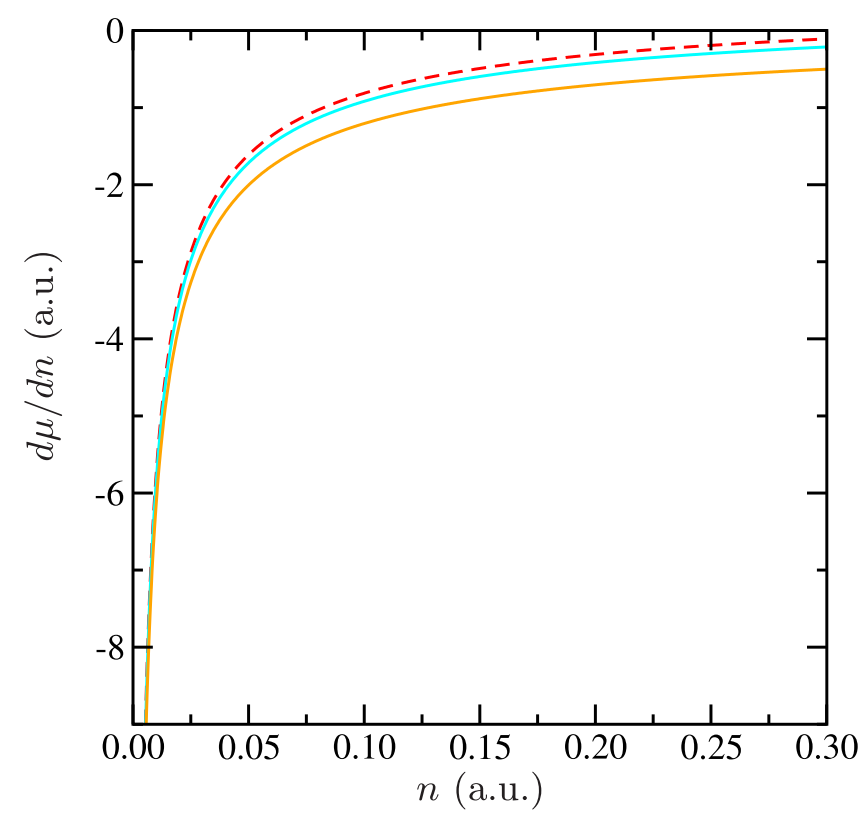

FIG. 5. Electronic compressibility as a function of the charge density including the Coulomb softening and the Hartree bandbending effects for quantum wells of different depths. The exchange is treated at the LDA level. The depth of the quantum well is infinite for the red-dashed line [same as in Fig. 4(a)], $250 \mathrm{Ha}$ for the cyan line, and $22 \mathrm{Ha}$ for the orange line. The width of the well is $w=$ 2.0 bohrs. All magnitudes in atomic units.

quantum wells (Sec. IV A), and (ii) the effect of the population of higher subbands (Sec. IV C).

\section{A. Traditional quantum well}

To validate our numerical implementation against the analytical results of the previous section, we consider, first of all, the "traditional quantum well" capacitor of Fig. 1 (referred to as $\mathrm{t}-\mathrm{QW}$ henceforth). Here the electrons in the quantum electrode are confined in a narrow layer by an external (usually large) potential $V_{\text {ext }}$, mimicking the conduction-band offsets at the QW boundaries. Within the setup discussed in this section we shall assume that $\rho_{\text {jell }}=0$ in Eq. (4), so the QW is charged with a charge density $n$, that is compensated by the charge density $-n$ located in the classical electrode. This configuration essentially corresponds to the model of Eisenstein and co-workers [13].

Figure 6 shows the evolution of the inverse capacitance density $\mathcal{C}^{-1}$, computed as in Eq. (8), and of the electronic compressibility ("Fermi level" contribution) $d \mu / d n$. [To remove any dependency on the geometry of the capacitor, we have substracted from $\mathcal{C}^{-1}$ a "geometrical capacitance" equal to $-4 \pi d / \kappa$, where $d$ is the distance between the classical plate and the center of the quantum well. Consistently, $z_{\mathrm{im}}$ will be referred to this specific feature of the quantum electrode, so we define $z_{\mathrm{im}}^{*}=z_{\mathrm{im}}-d$.] To make contact with the formalism of the previous section, we also show the analytical results for $d \mu / d n$, calculated by using the ground-state wave function of the finite well at $n=0$ as described in Sec. IIIC. As the effects of wave-function (WF) relaxation are neglected within the analytic model of Sec. III C, we shall refer to the dashed 


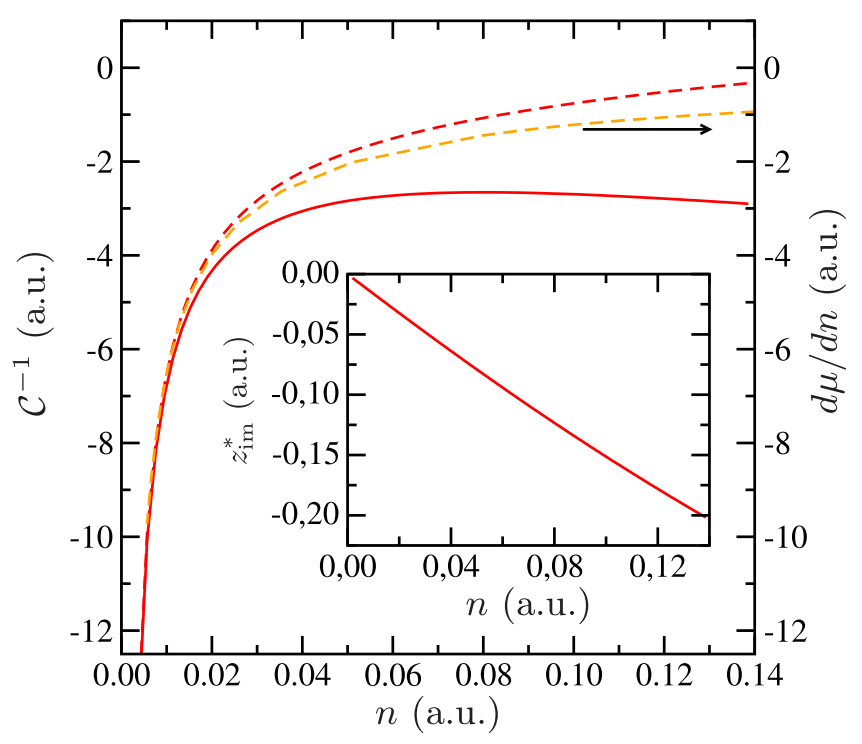

FIG. 6. Quantum contributions to $\mathcal{C}^{-1}$ in the traditional quantum well ( $\mathrm{t}-\mathrm{QW})$ model ( $w=2.0$ bohrs) as a function of the electronic density $n$. The solid red curve is the full relaxed-wave-function result. The Fermi level contribution [Eq. (31)] is represented by a dashed red curve. The dashed orange curve represents the electronic compressibility for the frozen wave-function results for a well of the same depth, $V_{\text {ext }}=-22$ Ha. Inset: evolution of $z_{\text {im }}^{*}$ (i.e., the image-plane location with respect to the geometric center of the QW) as a function of $n$.

orange curve as to the "frozen-WF" results. Recall that these values are always negative in the range of $n$ that we consider here, due to the combination of the thickened exchange (Sec. III B 1) and Hartree band-bending (Sec. III B 2) effects.

But, on top of these effects, the self-consistency on a QW of finite thickness introduces additional ingredients, whose impact on the physics was not clearly identified in earlier works [13]. Indeed, with respect to the frozen-WF result, the electronic compressibility as defined in Eq. (31) is slightly more positive, while the total inverse capacitance is significantly more negative. The difference between the latter two quantities consists in the "image-charge" contribution, reflected in the $4 \pi z_{\mathrm{im}} / \kappa$ term in Eq. (31), which is due to the displacement of the electronic charge density from the geometric center of the QW. Because of the electrostatic deformation of the electronic cloud, $z_{\mathrm{im}}$ is always smaller than the distance between the classical plate and the center of the QW, as shown in the inset of Fig. 6. This is, therefore, an extra source of negative capacitance that largely overcomes the small upward shift of $d \mu / d n$ with respect to the frozen-WF values. (The physical reasons behind both the overcompensation and the upward shift will be clarified in the next paragraphs.)

\section{B. Jellium slab with an external confinement potential}

For realistic simulations of many physical systems, it is convenient to treat the compensating charge as a uniform background within the quantum well itself, in the spirit of the jellium models [10]. In order to separate the effects introduced by charge compensation and confinement, we first carried out a set of simulations where we kept the same QW confining

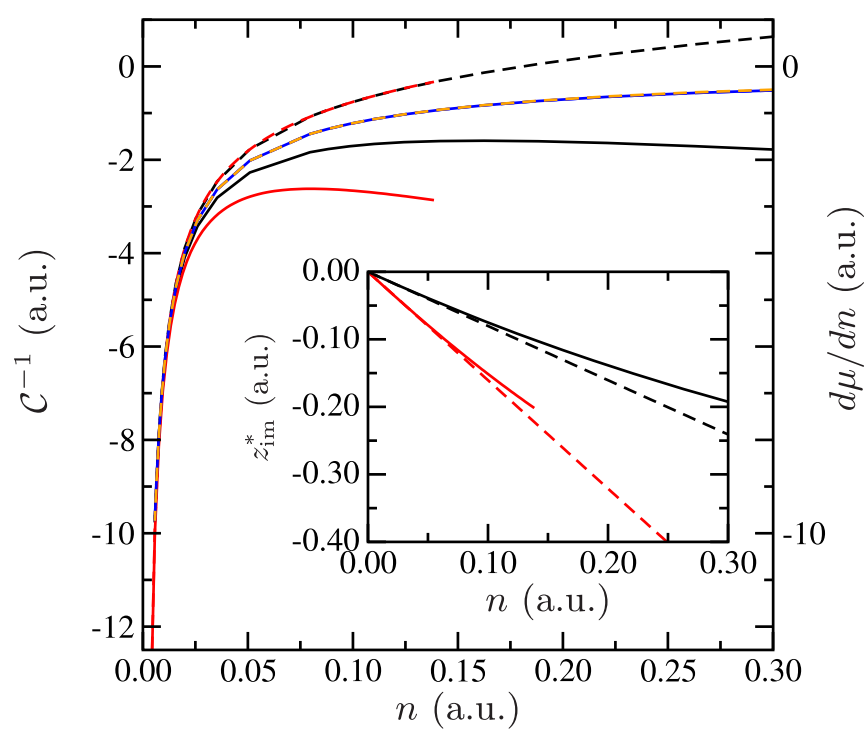

FIG. 7. Evolution of the inverse capacitance densities (solid lines; left $y$ axis) as a function of the charge density. The electronic compressibility contributions [Eq. (31)] are represented by dashed lines (right $y$ axis). Black lines represent the results of the confined jellium slabs where the electronic wave functions are allowed to relax. Blue lines are the results obtained when the electronic wave functions are frozen to the ones of a neutral jellium slab. Red lines represent the results of the traditional quantum well, already shown in Fig. 6, while the dashed orange line represents the analytical results for the electronic compressibility for a quantum well of the same thickness and depth and under the same LDA approach, already shown in Fig. 5. Inset: evolution of the position of the imagecharge plane (referred to $d$ as explained in the text) as a function of the charge density. Numerical results have been obtained for $m_{\|}=\kappa=1, w=2.0$ bohrs, $d=12.5$ bohrs, and $V_{\mathrm{ext}}=-22 \mathrm{Ha}$.

potential as in Sec. IV A, but added a jellium background $\rho_{\text {jell }}(z) \neq 0$ in Eq. (4), on top. The jellium density is defined as

$$
\rho_{\text {jell }}(z)=-n_{\text {jell }} W(z),
$$

where $W(z)$ is a "window function" equal to $1 / w$ inside the well and zero outside.

From a practical point of view, at every value of $n$ we shall first calculate the ground state of the neutral capacitor, by setting $n_{\text {jell }}=n$; the inverse capacitance density is then defined as the second derivative of the total energy with respect to $n$ at fixed $n_{\text {jell }}$.

The evolution of $\mathcal{C}^{-1}$, together with its decomposition into the electronic compressibility $(d \mu / d n)$ and image-plane $\left(z_{\text {im }}\right)$ contributions [Eq. (31)], as a function of the density in the jellium background, are shown as black curves in Fig. 7; for comparison, we also report the results of Fig. 6 for the bare quantum well as red curves. Remarkably, the evolution of the $d \mu / d n$ values with $n$ accurately match, regardless of whether we compensate the electronic charge with a jellium density (present case, j-QW henceforth) or not (traditional quantum well, t-QW).

Conversely, the total inverse capacitance density (inclusive of the image-plane contribution) is significantly more negative in the $\mathrm{t}-\mathrm{QW}$ case. To help explain these results, we follow 
a similar strategy as in the previous section and compute, in addition to the self-consistent results, the frozen-wavefunction (WF) compressibilities. [Here, at difference with the previous case where the $n=0$ wave function was used throughout, we calculate the "frozen-WF" result by using, at each $n$, the ground-state wave function of the neutral capacitor $\left(n=n_{\text {jell }}\right)$.] The results are plotted as a solid blue curve, and compared with the frozen-WF data of Fig. 6 (dashed orange); again, the two curves show an essentially perfect overlap.

Obviously, at $n=0$ the two curves must coincide, as the $\mathrm{j}-\mathrm{QW}$ model reduces to the $\mathrm{t}-\mathrm{QW}$ case in the limit of small densities. Deviations at finite $n$ can only stem from the deformation of the (symmetric) ground-state wave function due to the self-consistent potentials [for example, the superposition of $\rho_{\mathrm{el}}(z)$ and $\rho_{\mathrm{jell}}(z)$ produces a nonvanishing electrostatic potential whenever $n=n_{\text {jell }} \neq 0$ ]. Based on our result, we conclude that such deviations are negligible within our choice of geometry and computational parameters.

This latter observation allows us to interpret the combined effects of jellium compensation and wave-function relaxation (or lack thereof) in terms of an approximate model. In particular, for sufficiently small $\delta n$ we can assume, for the charged capacitor at $n=n_{\text {jell }}+\delta n$, that the relaxed wave function is

$$
\psi \sim \psi^{(0)}(n=0)+\delta n \psi^{(1)}(n=0) .
$$

Following a few derivation steps (see Appendix B), we could account for all the results presented so far. In particular, for both the t-QW and the j-QW models we find the same Hartree band-bending contribution

$$
\Delta_{\mathrm{H}}^{\text {relax }} \sim \Delta_{\mathrm{H}}^{\text {frozen }}+n z_{\mathrm{B}},
$$

where $z_{\mathrm{B}}$ is defined as the dipole moment of $\psi^{0}(z) \psi^{1}(z)$,

$$
z_{\mathrm{B}}=\int d z z \psi^{0}(z) \psi^{1}(z)
$$

Moreover, the value of $z_{\mathrm{im}}^{*}$ is twice as large in the t-QW case

$$
z_{\mathrm{im}}^{\mathrm{t}-\mathrm{QW}} \sim 4 n z_{\mathrm{B}}, \quad z_{\mathrm{im}}^{\mathrm{j}-\mathrm{QW}} \sim 2 n z_{\mathrm{B}},
$$

consistent with our self-consistent results (see inset of Fig. 7). (To corroborate this point we report, in the same inset, the predictions of the approximate model for $z_{\mathrm{im}}$.) Note that, within the approximations we used, the same quantity $z_{\mathrm{B}}$ is responsible for both the upshift of $d \mu / d n$ and the off centering of the electronic charge (image-plane effect).

The different behavior of $z_{\mathrm{im}}^{\mathrm{t}-\mathrm{QW}}$ and $z_{\mathrm{im}}^{\mathrm{j}-\mathrm{QW}}$, which constitutes the main result of this section, can be intuitively rationalized by observing the respective evolution of the ground-state and first-order electronic states with $n$. In the t-QW model, the ground-state electronic wave function is increasingly distorted for increasing $n$ since it is feeling a progressively stronger attraction by the classical electrode; conversely, in the j-QW case, the ground-state electronic wave function is always symmetric, and with a shape that is roughly insensitive to $n_{\text {jell }}$ [see Fig. 8(a)]. This effect propagates to the first-order densities [Fig. 8(b)], which in turn define $z_{\text {im }}$ following Eqs. (6) and (12). In particular, in the t-QW case, the distortion of $\psi(n)$ adds up to the contribution of the first-order wave functions, resulting in a $\rho_{\mathrm{el}}^{(1)}(z)$ whose off centering is twice as large compared to the $\mathrm{j}-\mathrm{QW}$ case.

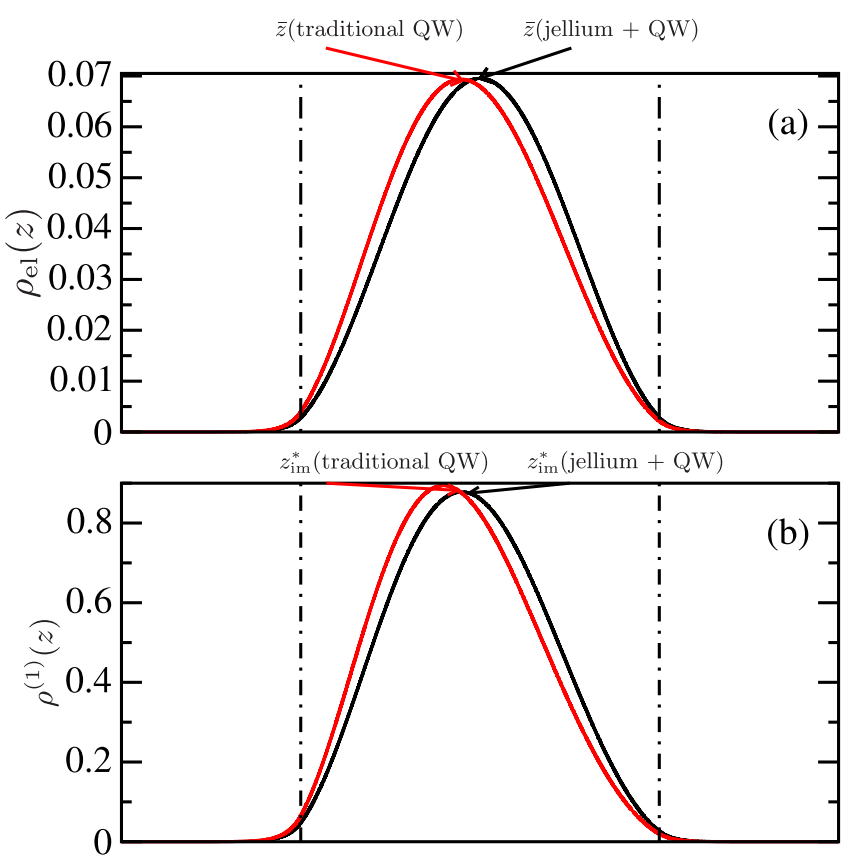

FIG. 8. (a) Electronic charge density profile of the confined jellium model in a neutral configuration (black solid line), and the traditional quantum well model (red solid line). (b) First-order variation of the electronic charge density in the two models. Vertical dotted-dashed lines represent the geometrical limits of the slab. The results have been obtained for a slab of $w=2.0$ bohrs, $m_{\|}=\kappa=1$, $n=0.079$ bohrs $^{-2}$, and $V_{\text {ext }}=-22 \mathrm{Ha}$. All magnitudes in atomic units.

Before concluding this part, we shall take the opportunity to corroborate, in light of the numerical results presented so far, an important point that we have already mentioned in Sec. II C: Due to the variational character of the problem, the relaxation of the wave functions always lowers the inverse capacitance density. This fact is immediately clear when we compare the relaxed (solid black line) and unrelaxed (solid blue line) results for $\mathcal{C}^{-1}$ in Fig. 7. As we have already emphasized, the electronic compressibility $d \mu / d n$ shows an opposite trend: the relaxed (dashed black curve) results are systematically more positive than the frozen-wave-function values (blue curve). This, however, is not in contradiction with the above argument: $d \mu / d n$, unlike $\mathcal{C}^{-1}$, cannot be written as a variational functional of $\psi^{(1)}$, and therefore is not bound to decrease upon relaxation.

\section{Jellium slab in the absence of strong confinement potential}

In Fig. 9 we plot the behavior of the inverse of the capacitance density as a function of $n$ for a 2.0-bohrs-thick jellium slab, this time without the confinement potential; this means that here the electron gas is only kept in place by the positive jellium background. As before, we also show the Fermilevel contribution, the frozen-WF results, and the image-plane location (inset). Remarkably, $\mathcal{C}^{-1}$ is much more negative than in the confined case discussed in the previous section (note the difference in vertical scale compared, e.g., with Fig. 7). This fact is already clear at the frozen-WF level, and is further enhanced by an unusually large negative contribution from $z_{\mathrm{im}}$. 


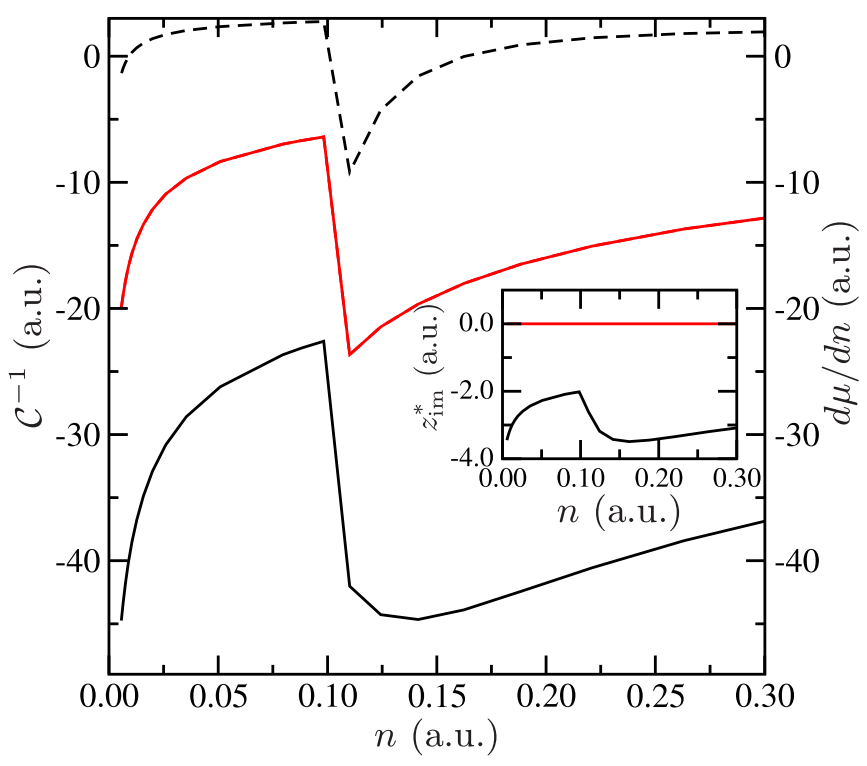

FIG. 9. Quantum contributions to $\mathcal{C}^{-1}$ in the jellium slab in the absence of a strong confinement potential (solid lines; left $y$ axis) as a function of the electronic density $n$. The solid black line is the fully relaxed wave-function result. The solid red line represents the results at the frozen-wave-function level. The black dashed line represents the electronic compressibility [Eq. (31)] (right $y$ axis). Inset: evolution of $z_{\mathrm{im}}^{*}$ as a function of $n$. Numerical results have been obtained for $m_{\|}=\kappa=1, w=2.0$ bohrs.

Moreover, at a density of about $n_{\text {crit }} \sim 0.10$ a.u. we observe a dramatic dip in both $\mathcal{C}^{-1}$ and $d \mu / d n$; such discontinuity, as we shall see shortly, is due to the transition from the one-band to the two-band regime. (Because of the weaker confinement, the energy separation between the different subbands is much smaller than in the previous examples, and multiple subbands may become occupied even at moderate values of the in-plane charge density.) We shall discuss these two regimes separately in the following.

\section{Low-density regime}

In the dilute regime (for low enough charge densities), only the lowest subband is occupied, as it happened in all the models analyzed up to now. The ground-state wave function, however, is now significantly more extended [Fig. 10(a)], and also more prone to be deformed when the capacitor is charged [Fig. 10(b)]; this implies that all the effects discussed in the previous sections are dramatically amplified.

At the frozen-WF level, as we pointed out in Sec. III B, the increase in the (negative) Hartree band-bending term (green line in Fig. 11) largely overcompensates for the reduction in the exchange contribution due to Coulomb softening (blue line in Fig. 11). This results in a drastic decrease in the electronic compressibility compared with a j-QW model of the same thickness.

Upon relaxation, while the electronic compressibility becomes positive, the overall inverse capacitance further decreases (solid black line in Fig. 9) compared to the frozen-WF case, in agreement with the behavior of $\mathrm{j}-\mathrm{QW}$ model. Interestingly, however, here $z_{\mathrm{im}}$ is substantially more shifted toward
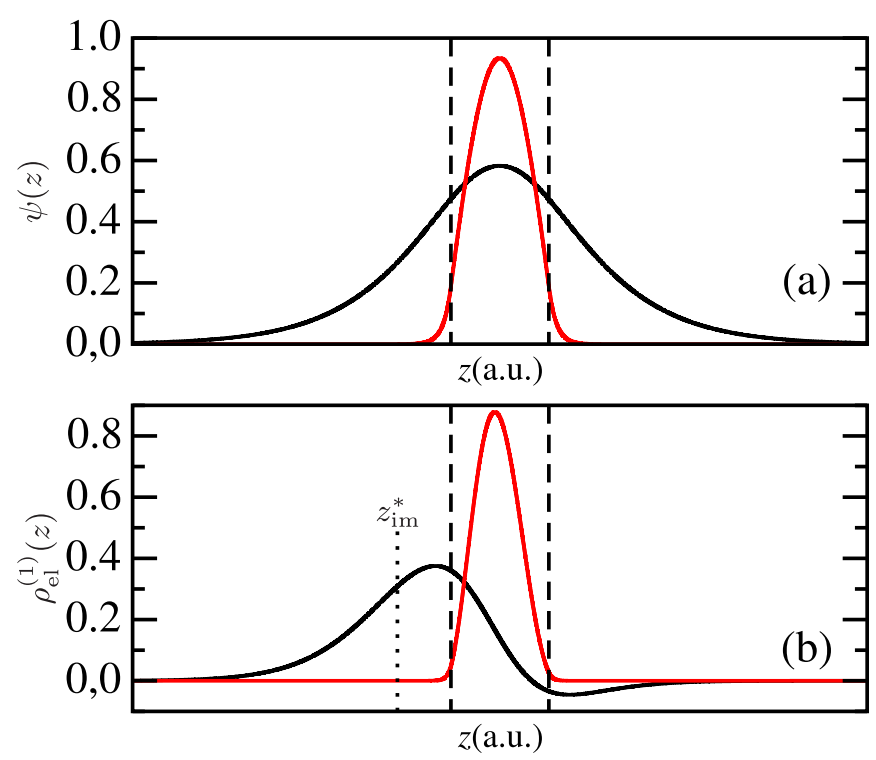

FIG. 10. (a) Real-space wave function of the lowest subband in a jellium slab without (black solid line) or with (red solid line) an extra confinement potential in the quantum well. (b) Profile of the total derivative of the self-consistent electronic charge density with and without the confinement potential. $z_{\mathrm{im}}^{*}$ represents the location of the image-charge plane, defined as in Eq. (12). The results have been obtained for a slab of $w=2.0$ bohrs, $m_{\|}=\kappa=1, V_{\text {ext }}=-22 \mathrm{Ha}$, $r_{\mathrm{s}}=2.0$. All magnitudes in atomic units.

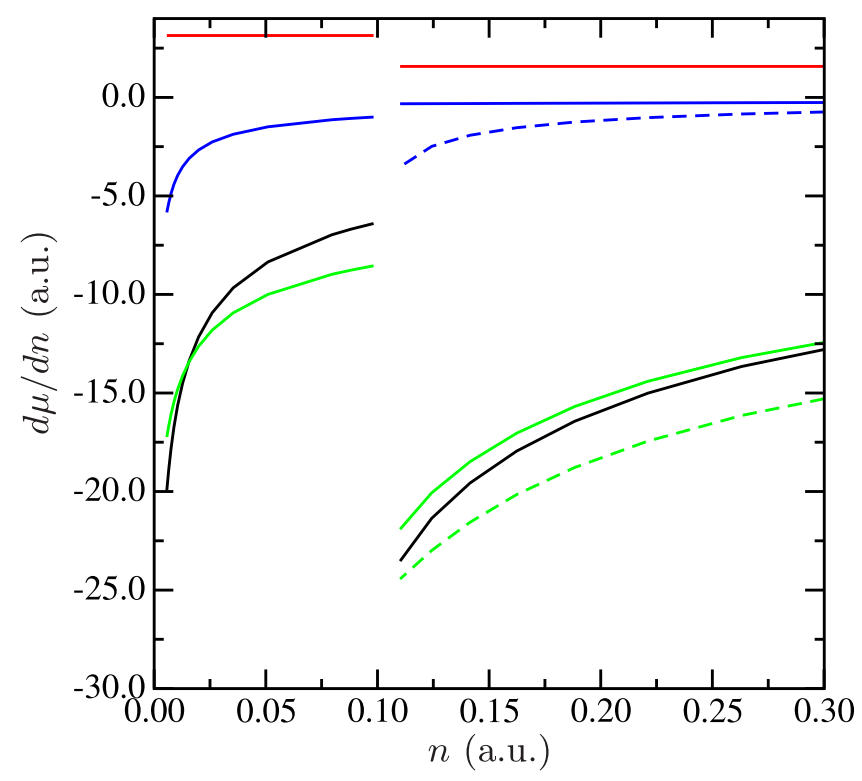

FIG. 11. Evolution of the electronic compressibility $d \mu / d n$ as a function of the charge density in the jellium slab in the absence of an external confinement potential (black line), and its decomposition into kinetic $\left(\pi / m_{\|} ;\right.$red), Hartree $\left[\Delta_{\mathrm{H}}^{\text {right }}(l)\right.$; green], and exchangecorrelation $\left[\Delta_{\mathrm{xc}}(l)\right.$; blue] contributions, where $l$ is an index for the occupied subband. The Hartree and exchange-correlation contributions coming from the first band are plotted in solid lines, while the contributions from the second band are represented by dashed lines. The electron wave functions are frozen to the ones obtained in a neutral slab $n=n_{\text {jell }}$. Results have been obtained for a slab of $w=2.0$ bohrs, $m_{\|}=\kappa=1$. 

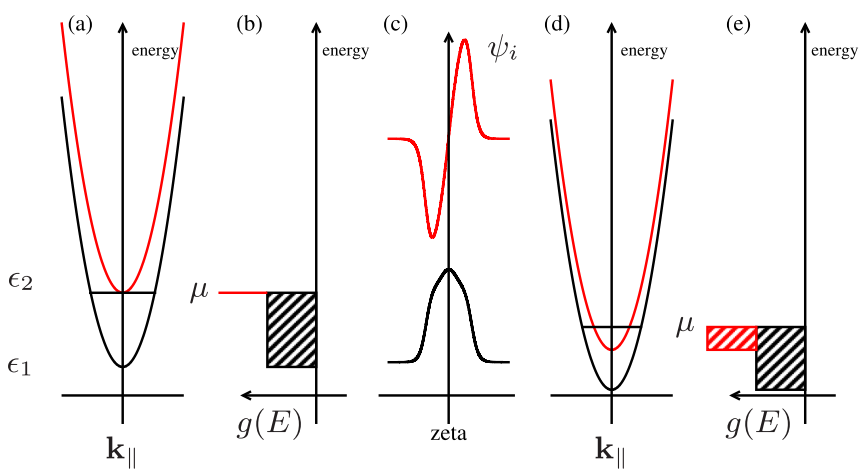

FIG. 12. (a) Energies of the first (black solid line) and second (red solid line) subbands as a function of the parallel $k$ point. (b) Contribution of the two subbands to the density of states $g(E)$ at the critical density when the energy of the highest occupied state in the first subband equals the eigenvalue of the second subband. (c) Radial shape of the eigenfunction of the one-dimensional Schrödinger equation along $z$ for the two subbands. Panels (d) and (e) are the same as (a) and (b), but for an increased charge density that produces the lowering of the chemical potential. Note how, despite the fact that the charge density is larger in (d) and (e) (larger occupied areas of the shaded rectangles), the chemical potential $\mu$ is lower than in (a) and (b).

the classical electrode, and follows a qualitatively different trend in the limit of small $n$. ( $z_{\text {im }}$ is a roughly linear function of $n$ in the j-QW case, while here it appears to diverge towards $-\infty$.) Both features, as we said, are due to the absence of a confining potential; this greatly enhances the charge-density response of the system to an external bias, and the more so in the dilute limit (the electrostatic attraction due to the jellium background vanishes for $n \rightarrow 0$ ).

\section{High-density regime}

Due to the absence of a confinement potential, there is a critical density [see the schematic illustration in Figs. 12(a) and 12(b)], where the Fermi level crosses the eigenvalue of the first excited subband. For larger densities, the two lowest subbands are partially occupied. As we have already demonstrated in Sec. II D, all contributions to the inverse capacitance are discontinuous at the transition. We shall illustrate them in detail hereafter, by framing our discussion around the frozenWF results (Fig. 11).

These provide a clearer insight on the underlying physics; on the other hand, wave-function relaxation does not introduce anything new that has not been discussed in the earlier paragraphs.

First, since we are considering that the in-plane effective masses are the same for all the bands, the in-plane kinetic energy term decreases by a factor of 2 at the transition (red segments in Fig. 11), and is otherwise constant within either regime. The remainder of $d \mu / d n$ originates from the variation with $n$ of the Hamiltonian eigenvalues, and can be further split into Hartree and exchange-correlation contributions of each occupied band. [In particular, one takes the weighted average of the contributions of all occupied bands, according to Eq. (49).]

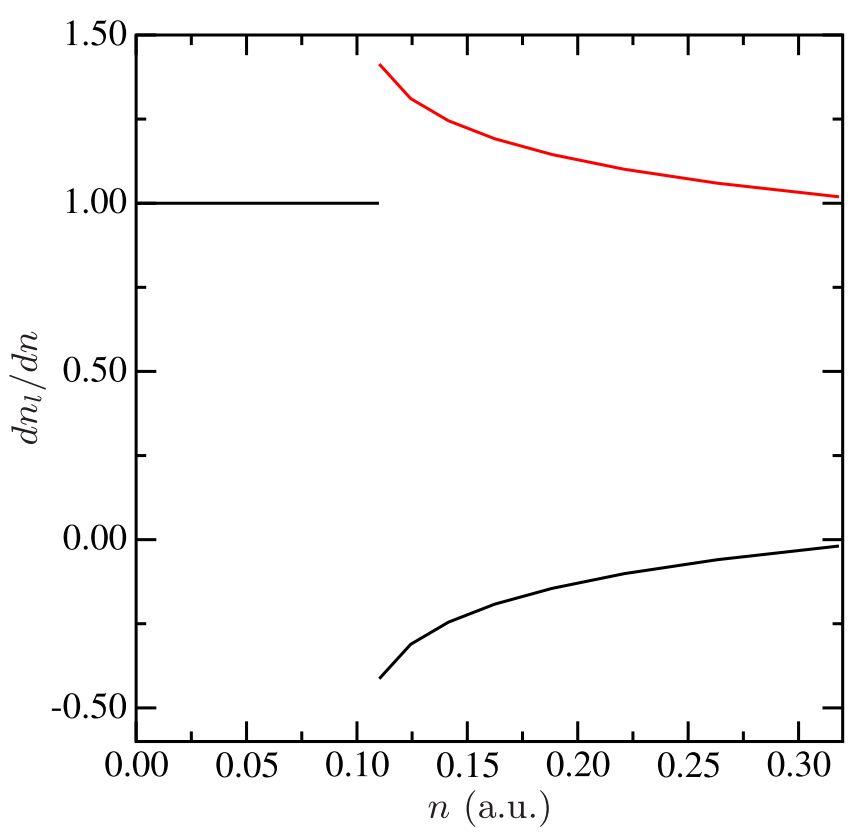

FIG. 13. Filling rate of the first (black line) and second (red line) subbands as a function of $n$ for a jellium slab of $w=2.0$ bohrs with frozen wave functions. (The individual curves are $d n_{l} / d n$, and must sum to unity as $\sum_{l} n_{l}=n$.)

As illustrated in Fig. 11, the drop of the electronic compressibility right after the critical density $n_{\text {crit }}$ is clearly dominated by the electrostatic contribution, which undergoes an abrupt drop at the transition. Interestingly, above $n_{\text {crit }}$ the Hartree contributions of both bands, $\Delta_{\mathrm{H}}^{\text {right }}(1)$ and $\Delta_{\mathrm{H}}^{\text {right }}(2)$, are equally large and negative. To see why, we need to look at the behavior of the first-order Hartree potential $\hat{V}_{\mathrm{H}}^{(1)}$ [recall Eq. (30)], which is in turn related to the first-order electronic density $\rho_{\mathrm{el}}^{(1)}(z)$ via a Poisson equation [Eq. (40)].

Below the transition $\rho_{\mathrm{el}}^{(1)}(z)$ corresponds the square modulus of the only occupied band

$$
\rho_{n<n_{\text {crit }}}^{(1)}(z)=\left|\psi_{1}(z)\right|^{2} .
$$

[Recall that we are working within the frozen-WF regime, which implies discarding the contribution of the first-order $\psi$ in Eq. (38).]

Conversely, above the transition we have

$$
\rho_{n>n_{\text {crit }}}^{(1)}(z)=\frac{d n_{1}}{d n}\left|\psi_{1}(z)\right|^{2}+\frac{d n_{2}}{d n}\left|\psi_{2}(z)\right|^{2},
$$

i.e., the first-order density (and, hence, the first-order potential) is now a linear combination of the squared moduli of the individual wave functions. (Note that the coefficients $d n_{l} / d n$ must sum up to 1 since $n=\sum_{l} n_{l}$.)

Remarkably, while $n_{l}$ are continuous functions of $n$ everywhere, their derivative is discontinuous at the transition. As shown in Fig. 13, right above $n_{\text {crit }}$ the filling rate of the first band becomes negative, while the second band hosts the entirety of the excess charge plus the amount that is being transferred from the first band. This abrupt change in the filling rate would have no effect whatsoever on the Hartree band bending if $\left|\psi_{2}(z)\right|^{2}$ were equal to $\left|\psi_{1}(z)\right|^{2}$. 


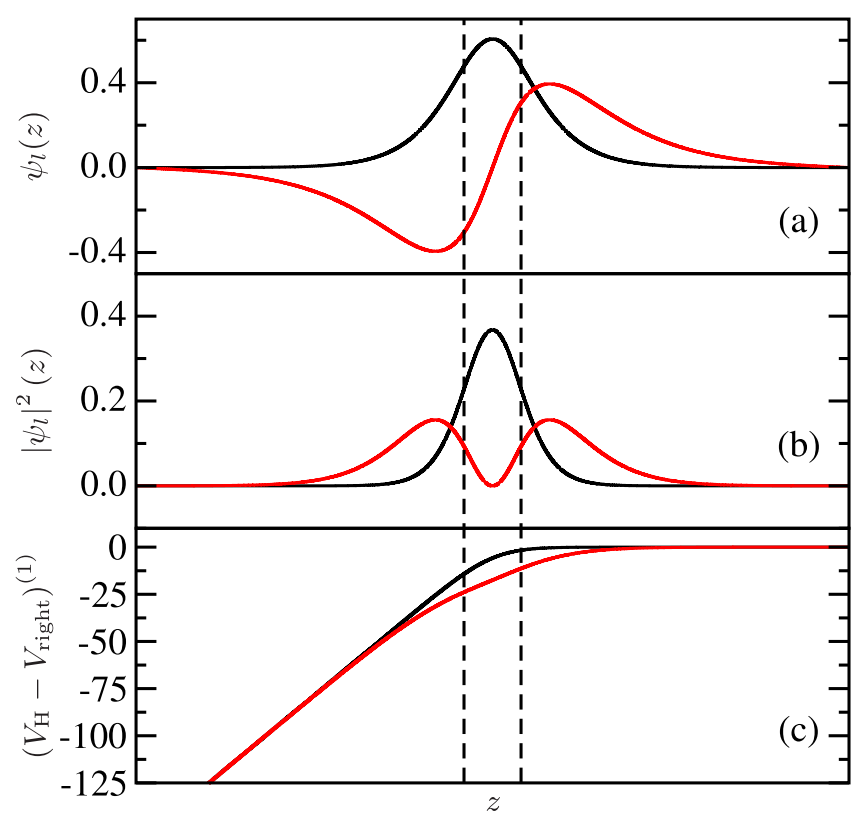

FIG. 14. (a) Real-space wave functions of the lowest-energy subband (black line) and the first excited state (red line). The corresponding contributions to the electronic charge density are shown in (b). (c) Profile of the first-order change in the electrostatic potential obtained by a double integral of $\left|\psi_{1}(z)\right|^{2}$ (black line) and $\left|\psi_{2}(z)\right|^{2}$ (red line). The electrostatic potential is measured with respect to the right, as this is the quantity that enters in the determination of the electronic compressibility. Dashed vertical lines mark the geometrical limits of the slab. The results have been obtained for a slab of $w=2.0$ bohrs, $m_{\|}=\kappa=1$, and $r_{\mathrm{s}}=1.7$. All magnitudes in atomic units.

The key point is that $\left|\psi_{2}(z)\right|^{2}$ is a much broader charge distribution than $\left|\psi_{1}(z)\right|^{2}$, as one can clearly see from Fig. 14(b). This means that $V^{(1)}(z)$, which is related to $\rho^{(1)}(z)$ via a double integration, will be significantly more negative for $n>n_{\text {crit }}$ than for $n<n_{\text {crit }}$ in a neighborhood of the jellium slab [see Fig. 14(c)]. As a consequence, both $\Delta_{\mathrm{H}}(1)$ and $\Delta_{\mathrm{H}}(2)$, defined as the mean value of $V^{(1)}(z)$ on the first and second eigenstates of the ground-state Hamiltonian, undergo an abrupt decrease at $n=n_{\text {crit }}$.

In other words, one can say that right at the transition the quantum electrode starts to accumulate charge into the second quantum state; this is spatially much broader than the lowest band, resulting in a greatly enhanced Hartree band-bending effect, consistent with what we have seen in all examples discussed so far (the thicker the electron gas, the more negative its compressibility).

This effect, of course, can be maintained only in a limited range of $n$ values. As the jellium charge density progressively increases, the electrostatic potential that keeps the electrons in place becomes deeper, the eigenfunctions shrink accordingly, and the Hartree band-bending effect becomes weaker. In fact, the same mechanism occurs in the dilute regime discussed above, where $\Delta_{\mathrm{H}}(1)$ shows an analogous monotonic increase with $n$. This is yet another consequence of the absence of a confining potential: Recall that in the j-QW model $\Delta_{\mathrm{H}}(1)$ is a constant at the frozen-WF level, as the ground-state wave functions undergo negligible changes with $n$.
Note that the same arguments are equally valid whenever a new band starts to be populated; however, we expect the enhancement of $d \mu / d n$ to become progressively smaller as the number of degrees of freedom increases (recall that the overall $d \mu / d n$ is written as a weighted average over all bands).

\section{Asymmetric confinement}

Since part of the renewed interest on negative compressibility is due to some recent experiments carried out in polar interfaces between two insulators $\left(\mathrm{SrTiO}_{3} / \mathrm{LaAlO}_{3}\right)$ [14], it is important to discuss, at least qualitatively, how the effects described in the previous sections manifest themselves in the latter context. As suggested in Ref. [21], the role of $\mathrm{LaAlO}_{3}$ is primarily to confine the conduction electrons to the $\mathrm{SrTiO}_{3}$ side, and define the electrostatic boundary conditions via an external surface charge density. This can be effectively modeled, within the methodology developed in this work, by using an asymmetric confining potential of Heaviside type, while controlling the electrical boundary conditions via the parameter $n$.

In addition, we shall include a thin jellium slab on the " $\mathrm{SrTiO}_{3}$ " side of the Heaviside potential, to guarantee a stable numerical solution. (As we shall see, the presence of the compensating jellium has little impact on the results at the qualitative level; it can be physically thought as a finite density of dopants that diffuse through the interface because of "intermixing.") This way, the electronic charge is confined only on one side of the slab, while it is free to relax on the other side. This asymmetry is clear in the spatial distribution of the ground-state electronic density [Fig. 15(a)]. The calculated behavior of the inverse capacitance density is shown in Fig. 15(b), together with the usual decomposition into Fermi-level and image-charge contributions.

Remarkably, the total $\mathcal{C}^{-1}$ undergoes a monotonic decrease with the charge density $n$, i.e., it shows an opposite trend than in all the situations analyzed up to now. This is clearly due to to the evolution of the image-charge-plane contribution, shown in the inset. (The electronic compressibility displays roughly the same behavior as the one described in Sec. IV C 2.) In the low-density regime, the presence of the Heaviside potential prevents the wave function from occupying the region between the two plates, while the weaker and weaker confining potential of the jellium lets the electronic density spread arbitrarily far to the other side (see Fig. 16). This translates into a divergence of $z_{\mathrm{im}}^{*}$ in the dilute limit, in stark contrast with the QW cases discussed earlier. At higher densities, higher subbands become occupied; their nodes lead then to oscillations in the first-order charge density (see red line in Fig. 16), effectively pushing its center of mass closer and closer to the electrode surface.

This effect, however, cannot overcome the surface confining barrier, which means that, in the limit of large $n, z_{\mathrm{im}}^{*}$ will slowly approach its asymptotic value of zero. Note that $z_{\mathrm{im}}^{*}$ (and hence the total inverse capacitance) is always positive in Fig. 15(b). This is mostly a matter of convention, though: Due to the geometry of the problem we find it more appropriate here to use the potential step as a reference to define the classical capacitance, unlike in the symmetrical QW cases of the previous sections. 


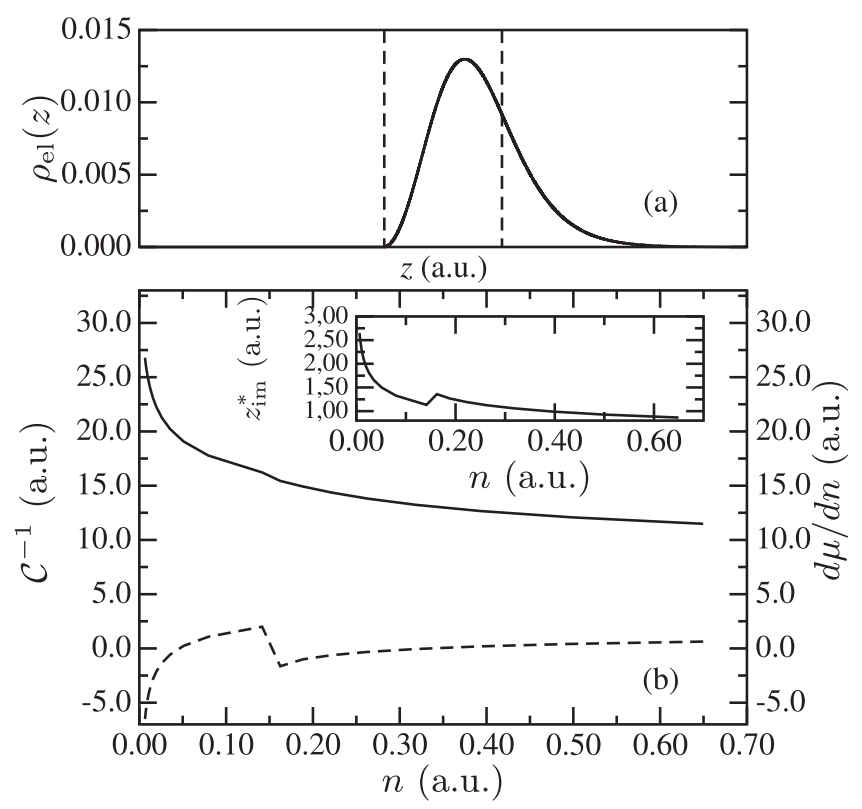

FIG. 15. (a) Electronic charge density in a jellium slab with a Heaviside step potential at the surface closer to the classical electrode. Vertical dashed lines represent the geometrical surfaces of the slab. The inverse capacitance density (solid line, left $y$ axis), and the electronic compressibility (dashed line, right $y$ axis) as a function of the charge density is shown in (b). Inset: position of the image-charge plane with respect to the geometrical center of the quantum electrode. The results have been obtained for a slab of $w=3.1$ bohrs of thickness, $r_{\mathrm{s}}=3.0[(\mathrm{a})]$, and $m_{\|}=\kappa=1$.

As we said above, the Fermi-level contribution qualitatively follows the same trend as in the cases discussed earlier. To substantiate this point, we show the breakdown of $d \mu / d n$

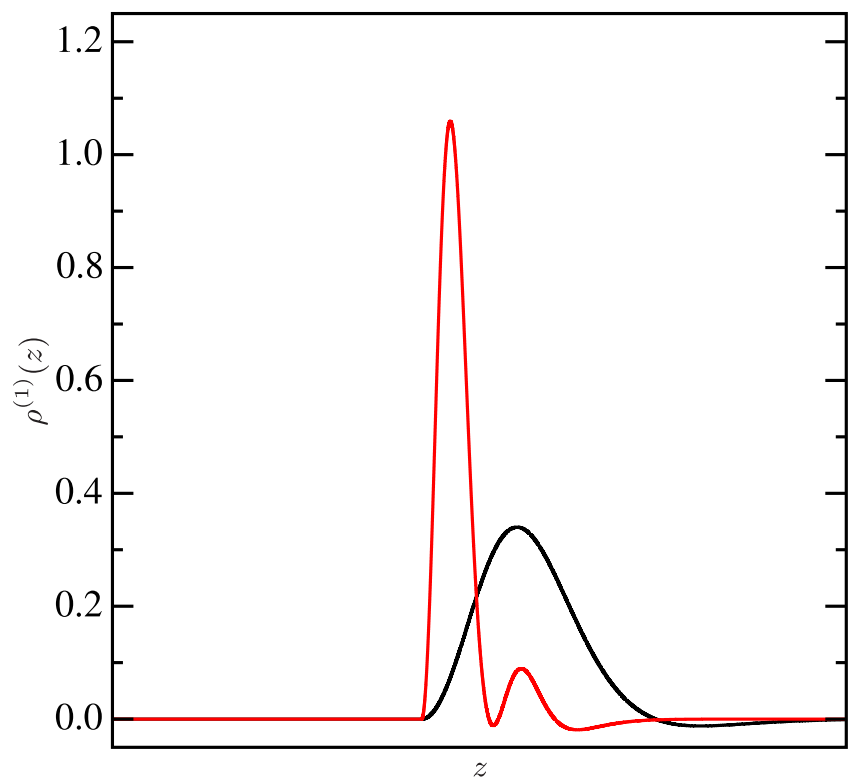

FIG. 16. Profile of the total derivative of the self-consistent electronic charge density in a jellium with an asymmetric confinement potential. Black line obtained for $r_{\mathrm{s}}=7.0$, and red line for $r_{\mathrm{s}}=0.7$. The rest of the parameters as in Fig. 15. All magnitudes in atomic units.

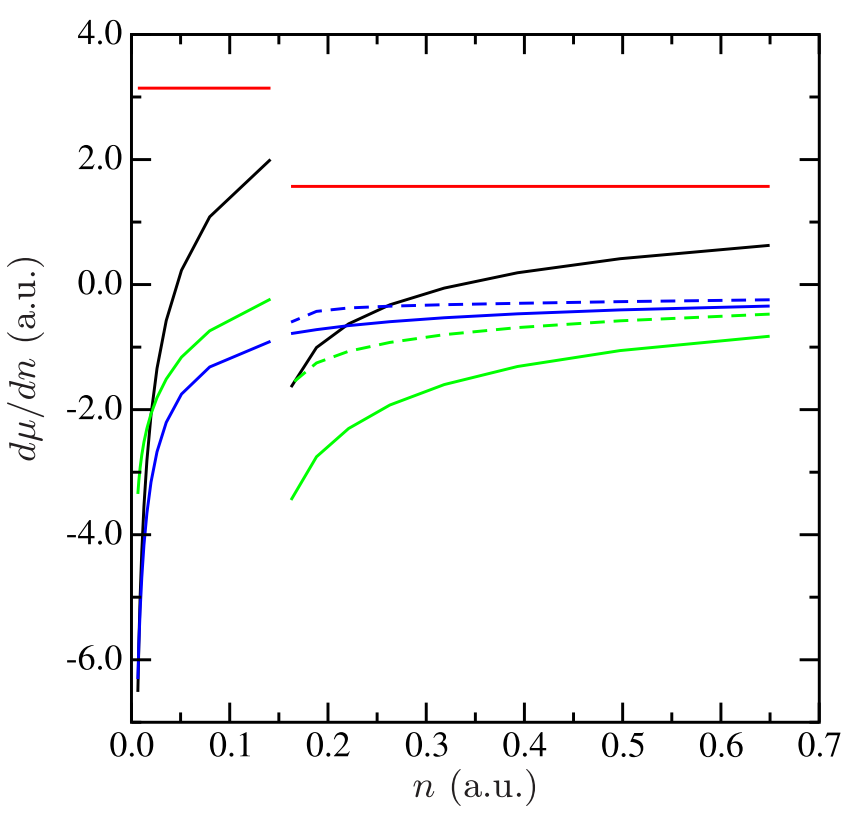

FIG. 17. Evolution of the electronic compressibility $d \mu / d n$ as a function of the charge density in the jellium slab with an asymmetric potential. Meaning of the lines as in Fig. 11. Results have been obtained for a slab of $w=3.1$ bohrs, and $m_{\|}=\kappa=1$. All magnitudes in atomic units.

into the individual contributions in Fig. 17. (This analysis is in all respects analogous to that of Fig. 11, with the sole difference that here we discuss the relaxed-wave-function values, rather than the frozen ones.)

As in Fig. 11, transition from the single- to multipleband regime produces a clear discontinuity, which is driven by a simultaneous reduction of the in-plane kinetic energy and a dip in the electrostatic Hartree band-bending terms. Interestingly, Fig. 17 shows an apparent reversal of the role of the bands above the transition. [In Fig. 11, $\Delta_{\mathrm{H}}(1)$ is always less negative than $\Delta_{H}(2)$, while here the opposite is true.] This should not be misinterpreted, though: Indeed, the upper band is still the primary responsible for the discontinuous change in $V_{\mathrm{H}}^{(1)}$ across the transition. Yet, $\Delta_{\mathrm{H}}(l)$ corresponds to the mean value of $V_{\mathrm{H}}^{(1)}$ on the $l$ th eigenfunction; the first band is spatially located closer to the surface, and hence can probe a region where $V_{\mathrm{H}}^{(1)}$ is deeper. In any case, the discontinuity almost disappears when the contributions of $z_{\mathrm{im}}^{*}$ and $d \mu / d n$ are summed up, leading to a simple monotonic behavior of $\mathcal{C}^{-1}$.

\section{DISCUSSION}

The fact that the inverse capacitance density is always positive in our asymmetric confinement model appears, at first sight, problematic in light of the experimental results of Refs. [14,15]. Our results also starkly disagree with the conclusions of Kopp and Mannhart [10] regarding the purported universality of the quantum capacitance effect. On the contrary, here we find a remarkable variability in the physical behavior of each individual system depending on geometry, confinement, and other factors. Interestingly, most of this variability is carried by two contributions that are 
electrostatic in nature: the Hartree band-bending and the image-charge effects. This result disproves earlier assumptions that exchange and kinetic effects would dominate [10], and prompts to a profound rethinking of the established interpretations.

Regarding the $\mathrm{LaAlO}_{3} / \mathrm{SrTiO}_{3}$ experiments [14,15], we regard it as highly unlikely that models based on the freeelectron gas such as those presented in this work (or in KM) will be able to explain the observed effects. Here, there are several indications that a more sophisticated description of electron correlations might be needed. For example, Ti $3 d$ orbitals in oxides are known [11] to form a two-dimensional electron liquid rather than an electron gas; strong electronic correlations may then localize the electrons and form polaronic quasiparticles. In this regime, the localized charges could couple with image charges in the other metal electrode and produce a dramatic increase of the capacitance as suggested by Skinner and Shklovskii [29]. (This model has been also recalled to explain the larger capacitances with respect to the classical values in gated carbon nanotubes [30] or in a black phosphorus thin film sandwiched between two layers of hexagonal boron nitride with a few-layer graphene as terminal electrodes [31].)

As we said, such scenarios are far beyond the range of applicability of our model, though. More accurate simulations, e.g., explicitly including the underlying atomic structure and the strong electron-electron interactions, would be desirable in the future to settle these important points.

Our predictions for a negative electronic compressibility that is associated with the population of higher subbands could be experimentally verified even in traditional semiconductor quantum wells (QWs). In fact, some of our results might be behind the first-order phase transition observed in $\mathrm{GaAs} / \mathrm{Al}_{x} \mathrm{Ga}_{1-x} \mathrm{QWs}$, when the first excited subband is occupied with electrons, as the Fermi level is tuned into resonance with the excited subband by applying a dc voltage [32]. In Ref. [32], the transition was attributed to a discontinuous jump of the exact 2D exchange potential every time a subband is occupied. A discontinuity in the exchange-correlation potential upon adding electrons to the ground state is, in principle, not expected within LDA; and indeed, in our calculations we observe a discontinuity in the derivative of the band occupations (and hence of the band eigenvalues) with respect to $n$, while the band eigenenergies are always continuous functions of $n$. At first sight, this seems to imply that exact exchange is crucial to obtaining a qualitative agreement with the experiments. However, we believe that this need not be the case, and that our results may even provide an alternative explanation to the experimental observations of Ref. [32]. The key point is that the voltage, rather than the electron density, is controlled in the experiments of Ref. [32]. We stress that, based on our results of Sec. IV C, the exchange energy and potentials are largely irrelevant in determining the quantum capacitance in the weak confinement limit. In such a regime, the physics is dominated by Hartree band-bending effects, which are electrostatic in nature. Based on the above arguments, the latter are therefore sufficient to produce a strong first-order transition as a function of the applied voltage when the Fermi level approaches the second subband.
Another remarkable feature of our simulations is a very large enhancement of the susceptibility in the negative capacitance regime. In a neutral jellium slab, the lowest and the first excited bands display opposite parities, and the total density is symmetric with respect the center of the slab. If an external electric field is applied, like the one produced by the classical plate after charging the slab, then the two bands can be hybridized, leading to an asymmetric electronic cloud as reflected by $z_{\mathrm{im}}$ in our simulations. This effect is particularly large when the first excited band starts filling and the two bands are essentially degenerate: the hybridization is almost costless and yields a large change in the dipole, that could be measured as a huge enhancement of the susceptibility of the system. Recent experiments in ferroelectric superlattices support the idea that the presence of regions with very large susceptibilities, like interfaces and domain walls [9,33,34], are fundamental to understand the boost of the capacitance in these systems.

Also, the aforementioned systems might be a perfect playground to check the role played by the effective mass or the dielectric constant of the medium in the description of real physical systems. It seems unlikely to us that the contribution of the valence electrons to the many-body interactions within the gas of carriers can be summarized by a single number (the effective dielectric constant). Even more, assuming that this approximation is valid, one can wonder what dielectric constant should be used: the static (including the response of the underlying lattice), or the high frequency (taking into account only the electronic response). While the ions should not mediate the exchange and correlation effects between the electrons, the use of the static dielectric constant has been the common approach in the semiconductor community. Such an approach appears problematic, however, in oxides, where the static and the high-frequency dielectric constants may differ by orders of magnitude.

As the dielectric constant appears at the denominator in the Hartree and exchange-correlation energies, then all the useful (old and new) mechanisms that we discussed in this work would be suppressed and the system would behave like a free-electron gas.

An insight on these issues would require the use of sophisticated many-body techniques; we regard it as an interesting topic for future studies.

\section{CONCLUSIONS}

Since the milestone works in semiconductor quantum wells aimed to understand the quantum Hall effect [13], the model based on the competition of the kinetic energy with the quantum exchange energy in the electron-electron interactions has been pointed out as the root of the negative electronic compressibility in two-dimensional metals. These have been applied even in systems as different as two-dimensional electron gases at oxide interfaces [14].

In this work we have proven how they must be taken with care when other systems are studied, such as delta-doping layers or two-dimensional metals where the confinement of the electrons to the well might be much smaller than in the previous systems. In such a situation, the kinetic and the exchangecorrelation effects are not the only pieces that are important, 
but other actors enter into play such as the Hartree interactions between more extended electron systems, the population of subbands of increasing energy, or the displacement of the center of charge of the electronic clouds that tend to decrease the effective distance between the plates of the capacitor. We have quantified all of them in a step-by-step basis using a jellium slab as a toy model. Exploiting the quantum nature of the metallic electrodes to overcome the classical limits on capacitor performance appears as a promising research avenue. The results reported here open the door to the rational design of devices based on negative electronic compressibility and related effects.

\section{ACKNOWLEDGMENTS}

We acknowledge P. de Castro-Manzano, J. Mannhart, and Th. Kopp for useful discussions. This work was supported by the Spanish Ministry of Economy and Competitiveness through the MINECO Grants No. FIS2012-37549-C05-04 and No. FIS2015-64886-394-C5-2-P, and P.G.-F. acknowledges support from Ramón y Cajal Grant No. RyC-201312515. M.S. acknowledges the support of MINECO Grants No. MAT2016-77100-C2-2-P and No. SEV-2015-0496, and of Generalitat de Catalunya (Grant No. 2017 SGR1506). This project has received funding from the European Research Council (ERC) under the European Unions Horizon 2020 research and innovation program (Grant Agreement No. 724529).

\section{APPENDIX A: FORM FACTOR WITHIN THE LOCAL DENSITY APPROXIMATION}

Considering Slater's expression for the exchange energy of the homogeneous electron gas $[35,36]$, then it can be written in a local form, which in atomic units reads as

$$
\epsilon_{x}^{\mathrm{LDA}}(\rho)=-\frac{3}{4 \kappa}\left(\frac{3}{\pi}\right)^{\frac{1}{3}} \rho^{\frac{1}{3}}
$$

where $\kappa$ is the relative permittivity of the medium. The exchange energy is then given by the three-dimensional integral

$$
E_{x}^{\mathrm{LDA}}=\int d^{3} r \epsilon_{x}\left[\rho_{\mathrm{el}}(\vec{r})\right] \rho_{\mathrm{el}}(\vec{r})=-\frac{3}{4 \kappa}\left(\frac{3}{\pi}\right)^{\frac{1}{3}} \int d^{3} r \rho_{\mathrm{el}}^{\frac{4}{3}}(\vec{r})
$$

The exchange energy per unit area for the particular case under study (square well wave function) can be written as

$$
E_{x}^{\mathrm{LDA}} / S=-\frac{3}{4 \kappa}\left(\frac{3}{\pi}\right)^{\frac{1}{3}}\left(\frac{2 n}{w}\right)^{\frac{4}{3}} \int_{0}^{w} d z \sin ^{\frac{8}{3}}(\pi z / w) .
$$

This can be, in turn, further simplified to

$$
E_{x}^{\mathrm{LDA}} / S=-\frac{3}{4 \kappa}\left(\frac{3}{\pi}\right)^{\frac{1}{3}}\left(\frac{2 n}{w}\right)^{\frac{4}{3}} \frac{w}{\pi} C
$$

where the constant $C$ can be calculated numerically as

$$
C=\int_{0}^{\pi} d z \sin ^{\frac{8}{3}}(z)=1.4003141 .
$$

Finally, we obtain the exchange energy per electron in the confined 2D gas,

$$
\epsilon_{x}^{\mathrm{LDA}}(n, w)=-\frac{3 C}{2 \pi \kappa}\left(\frac{6}{\pi}\right)^{\frac{1}{3}}\left(\frac{n}{w}\right)^{\frac{1}{3}} .
$$

Remarkably, just like in the case of the exact treatment, we can write $\epsilon_{x}^{\mathrm{LDA}}(n, w)$ as the "ideal" 2D exchange energy times a form factor

$$
\epsilon_{x}^{\mathrm{LDA}}(n, w)=\epsilon_{x}^{2 \mathrm{D}}(n) F^{\mathrm{LDA}}(\zeta)
$$

The explicit form factor is

$$
\begin{aligned}
F^{\mathrm{LDA}}(\zeta) & =\frac{3 C}{2 \pi \kappa}\left(\frac{6}{\pi}\right)^{\frac{1}{3}} \frac{3 \kappa}{4} \sqrt{\frac{\pi}{2}} n^{-\frac{1}{2}}\left(\frac{n}{w}\right)^{\frac{1}{3}} \\
& =\frac{9 C}{8 \pi}\left(\frac{6}{\pi}\right)^{\frac{1}{3}} \sqrt{\frac{\pi}{2}} \pi^{\frac{1}{6}} \zeta^{-\frac{1}{3}} \\
& =A \zeta^{-\frac{1}{3}}
\end{aligned}
$$

where $A=0.9436555$ is a dimensionless constant.

\section{APPENDIX B: EFFECT OF THE RELAXATION OF THE WAVE FUNCTION}

In this Appendix we shall analyze the influence of the jellium background charge density in the position of the image charge and in the Hartree band-bending contribution to the electronic compressibility. For the remainder of this Appendix, we shall refer to all the physical quantities related with the traditional quantum well described in Sec. IV A with the subscript "TQW," while all the quantities related with the jellium model, Sec. IV B, will be labeled as "jell."

For the traditional quantum well, the ground state for any value of the charge density $n$ can be approached as

$$
\psi_{\mathrm{TQW}}^{(0)}(z ; n) \approx \psi^{(0)}(z ; n=0)+n \psi^{(1)}(z ; n=0) .
$$

Thus, we can write the electron density, up to second order in $n$, in terms of the wave function and its derivative computed at $n=0$,

$$
\begin{aligned}
\rho_{\mathrm{TQW}, \mathrm{el}}^{(0)}(z ; n)= & n\left|\psi_{\mathrm{TQW}}^{(0)}(z ; n)\right|^{2}=n\left|\psi^{(0)}(z ; n=0)\right|^{2} \\
& +2 n^{2}\left|\psi^{(0)}(z ; n=0) \psi^{(1)}(z ; n=0)\right|,
\end{aligned}
$$

and, straightforwardly, the first-order variation of the electronic charge density

$$
\begin{aligned}
\rho_{\mathrm{TQW}, \mathrm{el}}^{(1)}(z ; n)= & \left|\psi^{(0)}(z ; n=0)\right|^{2} \\
& +4 n\left|\psi^{(0)}(z ; n=0) \psi^{(1)}(z ; n=0)\right| .
\end{aligned}
$$

Now, we switch our attention to the jellium case. The most important difference with respect the previous situation is that we have a dependency with respect to two parameters: $n$ and $n_{\text {jell }}$. Now, it can be assumed that the ground state and its firstorder derivative are not significantly modified when the charge density in the jellium changes,

$$
\psi_{\text {jell }}^{(0)}\left(z ; n_{\text {jell }}, n\right) \approx \psi_{\text {jell }}^{(0)}\left(z ; n_{\text {jell }}=0, n=0\right)=\psi^{(0)}(z ; n=0),
$$

$\psi_{\text {jell }}^{(1)}\left(z ; n_{\text {jell }}, n\right) \approx \psi_{\text {jell }}^{(1)}\left(z ; n_{\text {jell }}=0, n=0\right)=\psi^{(1)}(z ; n=0)$. 


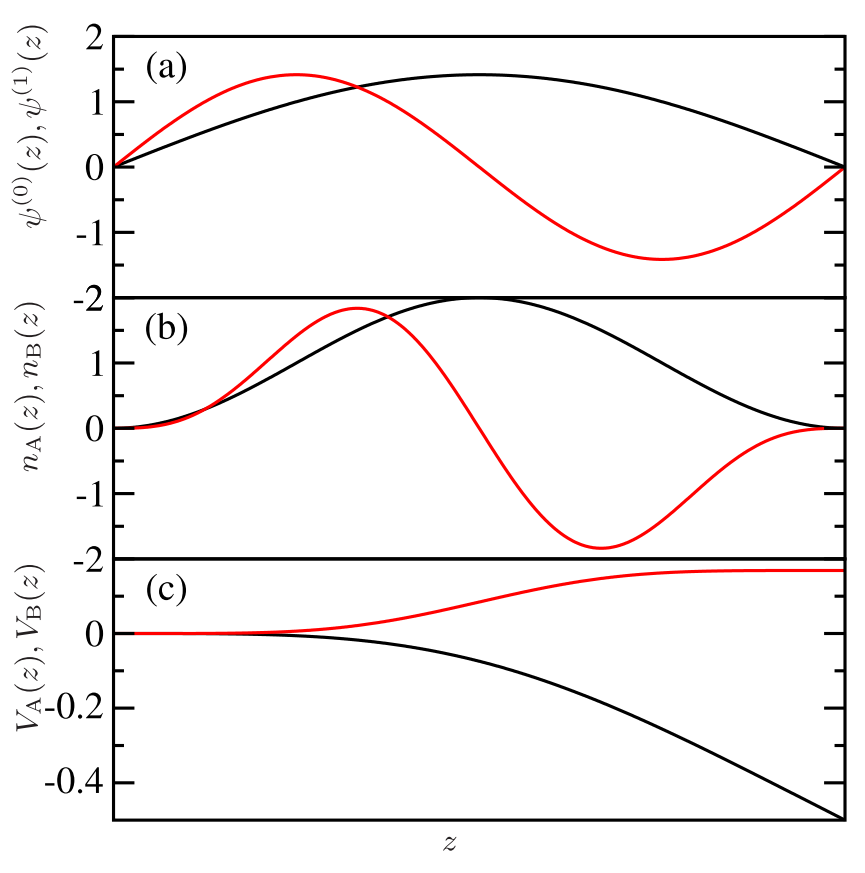

FIG. 18. (a) Ground-state (black line) and first-excited (red line) electronic eigenfunctions of a particle in an infinite quantum well potential in one dimension. (b) Associated $n_{\mathrm{A}}$ (black) and $n_{\mathrm{B}}$ (red) potentials as defined in the text. (c) Electrostatic potentials computed after a double integral of $n_{\mathrm{A}}\left(V_{\mathrm{A}}\right.$; black line) and $n_{\mathrm{B}}\left(V_{\mathrm{B}}\right.$; red line $)$.

We stress at this point that the ground state and first-order wave functions are exactly the same for the traditional quantum well and the jellium when $n_{\text {jell }}=0$ and $n=0$. The approach comes when we assume that they remain the same for any density.

Starting from the previous wave functions, the electron charge density can be computed in the jellium setup as

$$
\begin{aligned}
\rho_{\text {jell,el }}^{(0)}\left(z ; n_{\text {jell }}, n\right) & =n\left|\psi_{\text {jell }}^{(0)}\left(z ; n_{\text {jell }}, n\right)\right|^{2} \\
& \approx n\left|\psi^{(0)}(z ; n=0)\right|^{2} .
\end{aligned}
$$

For the first-order change in the charge density, we realize that in the process of charging/discharging the capacitor the variable that enters into play is $n$, while the background charge density $n_{\text {jell }}$ remains constant. Therefore, we have to take the partial derivative with respect to $n$, keeping $n_{\text {jell }}$ frozen:

$$
\begin{aligned}
\rho_{\text {jell,el }}^{(1)}\left(z ; n_{\text {jell }}, n\right)= & \left.\frac{\partial \rho_{\text {jell, }, \mathrm{el}}^{(0)}\left(z ; n_{\text {jell }}, n\right)}{\partial n}\right|_{n_{\text {jell }}}=\left|\psi^{(0)}(z ; n=0)\right|^{2} \\
& +2 n\left|\psi^{(0)}(z ; n=0) \psi^{(1)}(z ; n=0)\right| . \quad(\mathrm{B} 7)
\end{aligned}
$$

Now, and for the sake of simplicity, we shall revolve around a simplified version of the problem, based on the infinite quantum well, that captures the most important physical ingredients. From now on, we assume that $\psi^{(0)}$ and $\psi^{(1)}$ are the ground and first excited states of an infinite square quantum well. As shown in Fig. 18(a), these functions are orthonormal and, respectively, symmetric and antisymmetric with respect to the center of the quantum well. From these wave functions, we can compute the related densities $n_{\mathrm{A}}$ and $n_{\mathrm{B}}$, defined as

$$
\begin{aligned}
& n_{\mathrm{A}}(z)=\left|\psi^{(0)}(z)\right|^{2}, \\
& n_{\mathrm{B}}(z)=\psi^{(0)}(z) \psi^{(1)}(z),
\end{aligned}
$$

and plotted in Fig. 18(b). We can use these two densities to approximate the first-order changes in the charge densities for the traditional quantum well [Eq. (B3)], and the jellium model [Eq. (B7)]

$$
\begin{aligned}
\rho_{\mathrm{TQW}, \mathrm{el}}^{(1)}(z) & =n_{\mathrm{A}}(z)+4 n n_{\mathrm{B}}(z), \\
\rho_{\text {jell,el }}^{(1)}(z) & =n_{\mathrm{A}}(z)+2 n n_{\mathrm{B}}(z) .
\end{aligned}
$$

From the previous two equations we can immediately compute the position of the image charge of the two models, following Eqs. (6) and (12), as

$$
z_{\mathrm{im}}=\int d z z \rho^{(1)}(z)
$$

so, making use of the symmetry of the charge densities,

$$
\begin{aligned}
z_{\mathrm{im}}^{\mathrm{TQW}} & =4 n z_{B}, \\
z_{\mathrm{im}}^{\mathrm{jell}} & =2 n z_{B},
\end{aligned}
$$

where $z_{B}$ is the first moment of the charge distribution given by $n_{\mathrm{B}}$,

$$
z_{B}=\int z n_{\mathrm{B}}(z) d z
$$

Integrating the charge densities $n_{\mathrm{A}}$ and $n_{\mathrm{B}}$ we obtain two different potentials, coined in Fig. 18(c) as $V_{\mathrm{A}}$ and $V_{\mathrm{B}}$, respectively. For the sake of simplicity, in this simplified model we have assumed that the classical electrode is located at the right of the quantum well, i.e., the opposite convention as used in the rest of the work. This local change in the convention does not affect the conclusions that can be drawn from the model. The offset in the potential for $V_{\mathrm{B}}$ corresponds to the dipole moment associated with $n_{\mathrm{B}}$, as can be proven by an integration by parts,

$$
\Delta V_{\mathrm{B}}=V_{\mathrm{B}}(+\infty)-V_{\mathrm{B}}(-\infty)=4 \pi z_{B} .
$$

To compute the Hartree contributions to the electronic compressibility $d \mu / d n$ [Eq. (30)],

$$
\begin{aligned}
\Delta \epsilon_{\mathrm{H}, \mathrm{TQW}}^{(1)}(n) & =\int d z V_{\mathrm{H}, \mathrm{TQW}}^{(1)}(z ; n)\left|\psi_{\mathrm{TQW}}(z ; n)\right|^{2}, \\
\Delta \epsilon_{\mathrm{H}, \mathrm{jell}}^{(1)}(n) & =\int d z V_{\mathrm{H}, \mathrm{jell}}^{(1)}(z ; n)\left|\psi_{\mathrm{jell}}(z ; n)\right|^{2},
\end{aligned}
$$

where we have used, according to Eqs. (B1) and (B4),

$$
\begin{aligned}
\psi_{\mathrm{TQW}}(z ; n) & =\psi^{(0)}(z ; n=0)+n \psi^{(1)}(z ; n=0), \\
\psi_{\text {jell }}(z ; n) & =\psi^{(0)}(z ; n=0),
\end{aligned}
$$

and the potentials are the double integrals of $\rho_{\mathrm{TQW}, \mathrm{el}}^{(1)}$ and $\rho_{\text {jell,el }}^{(1)}$, respectively [Eq. (40)]. Then, neglecting terms beyond 
TABLE I. Conversion factors to rescale the numerical results obtained in this work to a material with effective mass $m^{*}$ and relative dielectric constant $\kappa$.

\begin{tabular}{ll}
\hline \hline Quantity & Factor \\
\hline Length & $\kappa / m^{*}$ \\
Energy & $\mathrm{m}^{*} / \kappa^{2}$ \\
Areal density & $\left(\mathrm{m}^{*} / \kappa\right)^{2}$ \\
Electronic compressibility & $1 / \mathrm{m}^{*}$ \\
\hline \hline
\end{tabular}

second order in $n$,

$$
\begin{aligned}
\Delta \epsilon_{\mathrm{H}, \mathrm{TQW}}^{(1)} & =V_{\mathrm{A}} n_{\mathrm{A}}+4 n V_{\mathrm{B}} n_{\mathrm{A}}+2 n V_{\mathrm{A}} n_{\mathrm{B}}, \\
\Delta \epsilon_{\mathrm{H}, \mathrm{jell}}^{(1)} & =V_{\mathrm{A}} n_{\mathrm{A}}+2 n V_{\mathrm{B}} n_{\mathrm{A}},
\end{aligned}
$$

where we have used the shorthand notation

$$
V_{x} n_{y}=\int d z V_{x}(z) n_{y}(z) .
$$

Paying attention to the symmetry of the potentials $V_{\mathrm{A}}$ and $V_{\mathrm{B}}$ in Fig. 18(c), they can be written as

$$
\begin{aligned}
& V_{\mathrm{A}}(z)=-\frac{z}{2}+f_{\mathrm{S}}(z), \\
& V_{\mathrm{B}}(z)=\frac{z_{B}}{2}+f_{\mathrm{A}}(z),
\end{aligned}
$$

where $f_{\mathrm{S}}$ and $f_{\mathrm{A}}$ are two functions that are, respectively, symmetric and antisymmetric with respect to the change $z \rightarrow-z$. Since $n_{\mathrm{A}}$ is normalized to unity, then

$$
\begin{aligned}
& V_{\mathrm{A}} n_{\mathrm{B}}=-\frac{z_{B}}{2}, \\
& V_{\mathrm{B}} n_{\mathrm{A}}=\frac{z_{B}}{2} .
\end{aligned}
$$

So we arrive to the final conclusion

$$
\Delta \epsilon_{\mathrm{H}, \mathrm{TQW}}^{(1)}=\Delta \epsilon_{\mathrm{H}, \mathrm{jell}}^{(1)}=V_{\mathrm{A}} n_{\mathrm{A}}+z_{B} n
$$

The first term in Eq. (B20) is the Hartree band bending for frozen wave functions, while the second is a linear correction with $n$ that appears when the wave functions are allowed to relax. With a similar derivation it is easy to prove that combining $V_{\mathrm{H} \text {,jell }}^{(1)}$ with $\psi_{\mathrm{TQW}}$ the linear part cancels out.

\section{APPENDIX C: UNITS}

The results discussed in this work have been calculated assuming a relative permittivity $\kappa=1$ and an effective electron mass $m^{*}=1$. Nevertheless, they can be used to interpret experiments where the materials under study present a different

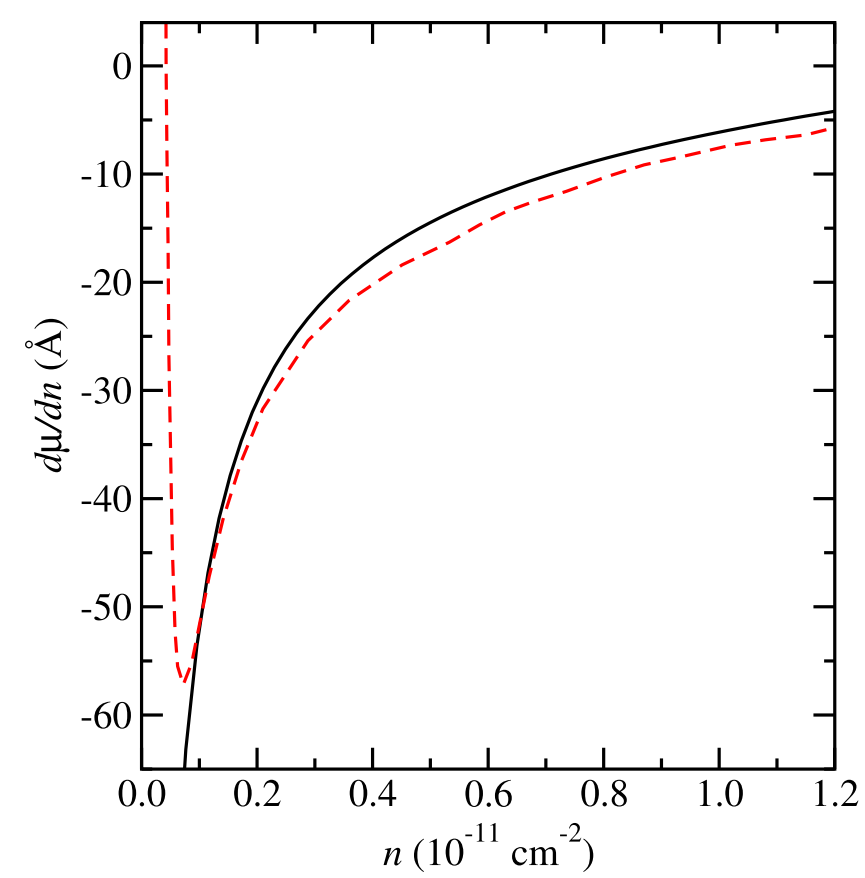

FIG. 19. Electronic compressibility as a function of electron density assuming the parameters of a realistic $\mathrm{GaAs} / \mathrm{Al}_{0.3} \mathrm{Ga}_{0.7} \mathrm{As}$ quantum well, $m^{*} / m_{\mathrm{e}}=0.067, \kappa=12.6 \epsilon_{0}$, and $V_{\text {ext }}=250 \mathrm{meV}$. The results are the same as in Fig. 6(b) but the physical magnitudes have been rescaled according to the rules given in Table I. Black solid line represents the theoretical results obtained for the traditional quantum well. Red dashed line is the experimental curve, taken from Ref. [13].

value of these two parameters. For this purpose, some physical quantities must be rescaled by the factors included in Table I.

Finally, note that the resulting $\partial \mu / \partial n$ is in atomic units of inverse capacitance density. To convert to a length (bohrs), this number needs to be rescaled by $\kappa / 4 \pi$. As an example, and as a convincing way to validate our calculations against experimental measurements, we have rescaled the results obtained in a simple quantum well potential to simulate the behavior of the electronic compressibility versus the charge density in $\mathrm{GaAs} / \mathrm{Al}_{x} \mathrm{Ga}_{1-x} \mathrm{As}$ quantum wells, as measured in Ref. [13]. Taking $V_{\text {ext }}$ to mimic the conduction-band offset of GaAs and $\mathrm{Al}_{0.3} \mathrm{Ga}_{0.7} \mathrm{As}$ (250 meV), assuming simple parabolic bands with effective mass $m^{*} / m_{e}=0.067$, and considering the dielectric constant to be $\kappa=12.6 \epsilon_{0}$ (where $\epsilon_{0}$ is the permittivity of free space), we obtain the results of Fig. 19 that compares very well with the reported experimental values. The theoretically predicted divergence of $d \mu / d n$ at low temperature is suppressed, presumably, by disorder in the low-density regime [13].
[1] G. E. Moore, Cramming more components onto integrated circuits, Reprinted from Electronics, volume 38, number 8, April 19, 1965, pp.114 ff, IEEE Solid-State Circuits Society Newsletter 11, 33 (2006).

[2] M. M. Waldrop, More than Moore, Nature (London) 530, 144 (2016).

[3] I. L. Markov, Limits on fundamental limits to computation, Nature (London) 512, 147 (2014).
[4] B. Sapoval and C. Hermann, Physics of Semiconductors (Springer, New York, 1993).

[5] R. Feynman, R. B. Leighton, and M. L. Sands, The Feynman Lectures on Physics, Volume II (Addison-Wesley, Reading, MA, 1964).

[6] S. Salahuddin and S. Datta, Use of negative capacitance to provide voltage amplification for low power nanoscale devices, Nano Lett. 8, 405 (2008). 
[7] A. Cano and D. Jiménez, Multidomain ferroelectricity as a limiting factor for voltage amplification in ferroelectric fieldeffect transistors, Appl. Phys. Lett. 97, 133509 (2010).

[8] W. Gao, A. Khan, X. Marti, C. Nelson, C. Serrao, J. Ravichandran, R. Ramesh, and S. Salahuddin, Roomtemperature negative capacitance in a ferroelectric-dielectric superlattice heterostructure, Nano Lett. 14, 5814 (2014).

[9] P. Zubko, J. C. Wojdeł, M. Hadjimichael, S. Fernandez-Pena, A. Sené, I. Luk'yanchuk, J.-M. Triscone, and J. Íñiguez, Negative capacitance in multidomain ferroelectric superlattices, Nature (London) 534, 524 (2016).

[10] T. Kopp and J. Mannhart, Calculation of the capacitances of conductors: Perspectives for the optimization of electronic devices, J. Appl. Phys. 106, 064504 (2009).

[11] J. Mannhart and D. G. Schlom, Oxide interfaces-an opportunity for electronics, Science 327, 1607 (2010).

[12] R. C. Ashoori and R. H. Silsbee, The Landau level density of states as a function of Fermi energy in the two dimensional electron gas, Solid State Commun. 81, 821 (1992).

[13] J. P. Eisenstein, L. N. Pfeiffer, and K. W. West, Compressibility of the two-dimensional electron gas: Measurements of the zero-field exchange energy and fractional quantum Hall gap, Phys. Rev. B 50, 1760 (1994).

[14] L. Li, C. Richter, S. Paetel, T. Kopp, J. Mannhart, and R. C. Ashoori, Very large capacitance enhancement in a twodimensional electron system, Science 332, 825 (2011).

[15] V. Tinkl, M. Breitschaft, C. Richter, and J. Mannhart, Large negative electronic compressibility of $\mathrm{LaAlO}_{3}-\mathrm{SrTiO}_{3}$ interfaces with ultrathin $\mathrm{LaAlO}_{3}$ layers, Phys. Rev. B 86, 075116 (2012).

[16] N. D. Lang and W. Kohn, Theory of metal surfaces: Induced surface charge and image potential, Phys. Rev. B 7, 3541 (1973).

[17] From now on, and on the sake of simplicity, we assume that there is no discontinuity in this parameter at the electrode/ dielectric interface, so the effective dielectric constant of the electrode is also $\kappa$.

[18] S. Baroni, S. de Gironcoli, A. D. Corso, and P. Giannozzi, Phonons and related crystal properties from density-functional perturbation theory, Rev. Mod. Phys. 73, 515 (2001).

[19] X. Gonze, Perturbation expansion of variational principles at arbitrary order, Phys. Rev. A 52, 1086 (1995).

[20] X. Gonze and C. Lee, Dynamical matrices, born effective charges, dielectric permittivity tensors, and interatomic force constants from density-functional perturbation theory, Phys. Rev. B 55, 10355 (1997).
[21] M. Stengel, First-Principles Modeling of Electrostatically Doped Perovskite Systems, Phys. Rev. Lett. 106, 136803 (2011).

[22] W. Kohn and L. J. Sham, Self-consistent equations including exchange and correlation effects, Phys. Rev. 140, A1133 (1965).

[23] D. M. Ceperley and B. J. Alder, Ground State of the Electron Gas by a Stochastic Method, Phys. Rev. Lett. 45, 566 (1980).

[24] P. Hohenberg and W. Kohn, Inhomogeneous electron gas, Phys. Rev. 136, B864 (1964).

[25] See the notes by P. Gianozzi at http://www.fisica.uniud.it/ giannozz/Corsi/MQ/LectureNotes/mq-cap1.pdf

[26] L. Kleinman, Comment on the average potential of a wigner solid, Phys. Rev. B 24, 7412 (1981).

[27] B. Tanatar and D. M. Ceperley, Ground state of the two-dimensional electron gas, Phys. Rev. B 39, 5005 (1989).

[28] F. Stern, Exchange energy in inversion layers: Thickness effects, Jpn. J. Appl. Phys. 13, 323 (1974).

[29] B. Skinner and B. I. Shklovskii, Anomalously large capacitance of a plane capacitor with a two-dimensional electron gas, Phys. Rev. B 82, 155111 (2010).

[30] H. Fu, B. I. Shklovskii, and B. Skinner, Correlation effects in the capacitance of a gated carbon nanotube, Phys. Rev. B 91, 155118 (2015).

[31] Y. Wu, X. Chen, Z. Wu, S. Xu, T. Han, J. Lin, B. Skinner, Y. Cai, Y. He, C. Cheng, and N. Wang, Negative compressibility in graphene-terminated black phosphorus heterostructures, Phys. Rev. B 93, 035455 (2016).

[32] A. R. Goñi, U. Haboeck, C. Thomsen, K. Eberl, F. A. Reboredo, C. R. Proetto, and F. Guinea, Exchange instability of the two-dimensional electron gas in semiconductor quantum wells, Phys. Rev. B 65, 121313(R) (2002).

[33] R. Landauer, Can capacitance be negative? Collect. Phenom. 2, 167 (1976).

[34] A. K. Yadav, K. X. Nguyen, Z. Hong, P. García-Fernández, P. Aguado-Puente, C. T. Nelson, S. Das, B. Prasad, D. Kwon, S. Cheema, A. I. Khan, C. Hu, J. Íñiguez, J. Junquera, L.-Q. Chen, D. A. Muller, R. Ramesh, and S. Salahuddin, Spatially resolved steady-state negative capacitance, Nature (London) 565, 468 (2019).

[35] P. A. M. Dirac, Note on the exchange phenomena in the Thomas atom, Proc. Cambridge Philos. Soc. 26, 376 (1930).

[36] J. C. Slater, A simplification of the Hartree-Fock method, Phys. Rev. 81, 385 (1951). 